\title{
Mid-Lift-to-Drag Ratio Rigid Vehicle Control System Design and Simulation for Human Mars Entry
}

\author{
Breanna J. Johnson ${ }^{1}$, Christopher J. Cerimele ${ }^{2}$, Susan J. Stachowiak ${ }^{3}$, Ronald R. Sostaric ${ }^{4}$, Daniel A. Matz ${ }^{5}$ \\ NASA Johnson Space Center, Houston, TX, 77058 \\ and \\ Ping $\mathrm{Lu}^{6}$ \\ San Diego State University, 5500 Campanile Drive, San Diego, CA 92182
}

The Mid-Lift-to-Drag Ratio Rigid Vehicle (MRV) is a proposed candidate in the NASA Evolvable Mars Campaign's (EMC) Pathfinder Entry, Descent, and Landing (EDL) architecture study. The purpose of the study is to design a mission and vehicle capable of transporting a 20mt payload to the surface of Mars. The MRV is unique in its rigid, asymmetrical lifting-body shape which enables a higher lift-to-drag ratio (L/D) than the typical robotic Mars entry capsule vehicles that carry much less mass. This paper presents the formulation and six-degree-of-freedom (6DOF) performance of the MRV's control system, which uses both aerosurfaces and a propulsive reaction control system (RCS) to affect longitudinal and lateral directional behavior.

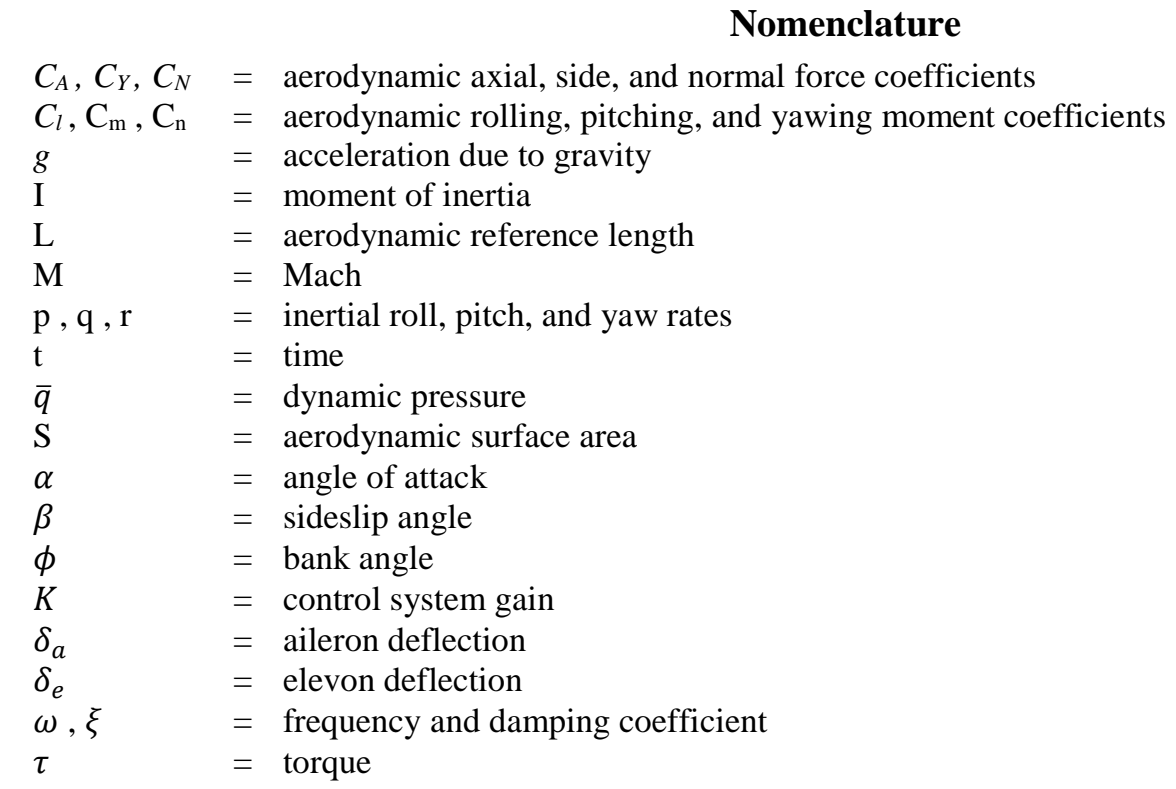

\footnotetext{
${ }^{1}$ Aerospace Engineer, Flight Mechanics and Trajectory Design Branch NASA JSC/EG5.

${ }^{2}$ EDL Domain Lead, Aeroscience and Flight Mechanics Division, NASA JSC/EG5.

${ }^{3}$ Aerospace Engineer, Flight Mechanics and Trajectory Design Branch NASA JSC/EG5.

${ }^{4}$ Aerospace Engineer, Flight Mechanics and Trajectory Design Branch NASA JSC/EG5, AIAA Senior Member.

${ }^{5}$ Aerospace Engineer, Flight Mechanics and Trajectory Design Branch NASA JSC/EG5, AIAA Senior Member.

${ }^{6}$ Professor, Department of Aerospace Engineering; plu@ @dsu.edu, Fellow AIAA.
} 


\section{Introduction}

$\mathrm{I}_{\mathrm{c}}^{\mathrm{N}}$ $\mathrm{N}$ an effort to expand human presence beyond near Earth operations, NASA has formulated the Evolvable Mars Campaign (EMC) to develop a strategy to define the capabilities needed for a Human Mars mission. As a result of this campaign, NASA has initiated a multi-center feasibility assessment, the Human Mars EDL Pathfinder study, to define the risks and benefits of candidate future vehicles that would be capable of delivering a 20 metric ton (mt) payload to the surface of Mars. ${ }^{1}$ This requirement stems from an estimated mass constraint for maximum loading of the Space Launch System (SLS) payload fairing and an ability to transport a Mars Ascent Vehicle (MAV) for a future human return mission. While NASA has landed a large payload of this approximate size on Earth with the Space Shuttle, this has not yet been achieved on Mars. With an average density equal to only about $1 \%$ of the Earth at sea level, the Mars atmosphere provides little aerobraking for vehicles toting the large payloads estimated to be needed for human settlement. In fact, the largest payload that has successfully landed on Mars is the Mars Science Laboratory (MSL) Curiosity rover which has a mass of slightly less than $1 \mathrm{mt}^{2}$

In order for a vehicle to successfully complete Entry, Descent, and Landing (EDL), it must demonstrate controllability for the duration of the EDL, whether through its inherent stability from design or via augmentation. To date, all fully successful Mars landings have been completed using a blunt-body, 70 deg sphere cone entry shape. A number of different shapes have been flown at high speed entry conditions $(\sim 7 \mathrm{~km} / \mathrm{s})$ at Earth. For human spaceflight, these are limited to blunt-body capsules ( $\mathrm{L} / \mathrm{D}<=0.3)$ and the high-L/D Space Shuttle (L/D =1.5). In order to land on the target runway, the Shuttle utilized aerosurfaces typically used on aircraft, such as ailerons, an elevator, and a rudder. In addition, it also had Reaction Control System (RCS) thrusters for attitude control. Capsules have utilized passive control with reliance on inherent stability as well as active control with reliance on RCS during entry as methods of attitude control. These capsules typically have used parachutes for their final descent phases. The MRV is an intermediate approach between the winged Shuttle and a blunt-body capsule. The vehicle takes advantage of launch vehicle shape for increased payload volume. Its control approach is similar to the Shuttle, but at a lower L/D $(\mathrm{L} / \mathrm{D}=0.57)$. The generated lift vector is controlled according to a guidance law that determines the guidance bank commands needed to At Mars, an extremely low ballistic coefficient is needed to slow a spacecraft to a subsonic condition. Large mass Mars landing vehicles are too heavy for any practical approach to parachutes to be of much benefit. As a result, powered flight must begin at a supersonic flight condition. The control approach described in this paper addresses the entry flight up to the start of the supersonic retro-propulsion (SRP) burn.

Once the MRV general capabilities and limitations had been established, the next step was to determine which class of attitude control actuators would best allow for precise handling with maximum fuel savings. The longitudinal as well as lateral directional controls would need to be designed for aerodynamic stability in response to perturbations to allow for natural damping and would benefit from a combination of aerosurfaces as well as RCS thrusters for precision EDL. In order to assess the feasibility of an MRV mission, the control system's ability to maintain its high angle of attack trim, reduce sideslip, and closely track guidance commands within a given deadband was evaluated. Due to the human crew and the possibility of a pilot input, there exists a need to evaluate the Dutch roll/short period frequencies and damping on the guidance and control system response. The development, results, and analysis of this blended jet and aero surface system is described below.

\section{Control System Design and Development}

The delivered control system was developed by using the JSC Flight Analysis Simulation Tool (FAST), to provide a 3-DOF/6-DOF simulation environment. Previous 3-DOF trajectories utilized perfect control, but there was no way to simulate an RCS thrust acceleration ${ }^{3}$ Because of this, a pseudo-1-DOF controller that emulates an applied bank acceleration and rate limit was created. Monte Carlo dispersions on angular accelerations and rate limits influenced RCS jet design and requirements. Jet locations were then determined and verified with later 6-DOF Monte Carlo analysis. Next the aerodynamic stability of the vehicle and the Military Specification (MILSPEC) for flying qualities of piloted airplanes were used to define control system minimum requirements. Once these parameters were defined, nonlinear equations of motion representative of the aerodynamic and applied torques were derived, then linearized about the aerodynamic stability trim conditions. These second order linear equations of motion are then used to derive the gains necessary to produce a proportional-derivative (PD) controller with a response within specified MILSPEC requirements for preferential handling. Step inputs were simulated in MATLAB to validate that the augmented frequencies and damping ratios are producing the intended system response. Once validation was completed, this control system was integrated into the FAST environment for a 6-DOF nominal trajectory with perfect state updates. Once these results proved satisfactory, the nominal trajectory entry inputs were dispersed in a Monte Carlo simulation to assess the performance and robustness of the control system. These results were evaluated by confirming maximum g-loads were within typical human safety requirements of less than 4 Earth g's and by how many results landed within 
$5 \mathrm{~km}$ of the target, which is also defined as the location at which input target energy conditions are met for powered descent initiation (PDI).

\section{II.A. RCS Jet Design}

After a nominal 3-DOF reference trajectory with perfect control had been established using the Fully Numerical Predictive-corrector Entry Guidance (FNPEG) ${ }^{4}$ an RCS jet sizing study was completed to establish the control system requirements, a process similar to what has been done for low L/D Aeromaneuvering Orbital Transfer Vehicles (AOTVs). ${ }^{5}$ This study used the reference trajectory with a pseudo-1-DOF controller simulates RCS jet firings by applying constant pitch or bank angular accelerations within given angular rate limits. Angular acceleration requirements can approximate the levels of thrust and number of RCS thrusters needed, while angular rate requirements impact RCS propellant usage. Minimal angular accelerations and rates were desired to decrease the number of RCS jets and tank masses, but the design angular acceleration also needed to be large enough to achieve adequate trajectory performance. Adequate performance was defined as achieving less than $5 \mathrm{~km}$ miss distance from the FNPEG target.

Due to the assumption that the MRV would naturally trim about a predetermined angle of attack due to inherent static stability, the study focused on sizing jets needed to follow the more challenging guidance bank commands from FNPEG. To simulate jet firings, an emulator applied a user-specified discrete bank acceleration, then calculated the resultant angular rate and bank angle based on a deadband, the maximum rate limit, and rotational kinematic equations:

$$
\begin{gathered}
\dot{\phi}=\dot{\phi}_{0}+\ddot{\phi}_{c} \Delta \mathrm{t} \\
\phi_{c}=\frac{1}{2} \ddot{\phi}_{c} \Delta t^{2}+\dot{\phi}_{0} \Delta \mathrm{t}+\phi_{0}
\end{gathered}
$$

Guidance-generated-bank commands from a 3-DOF trajectory with perfect control were used to test emulator performance before integration into the FAST environment, and an example of its results for different rate limits are shown in Figure 1.

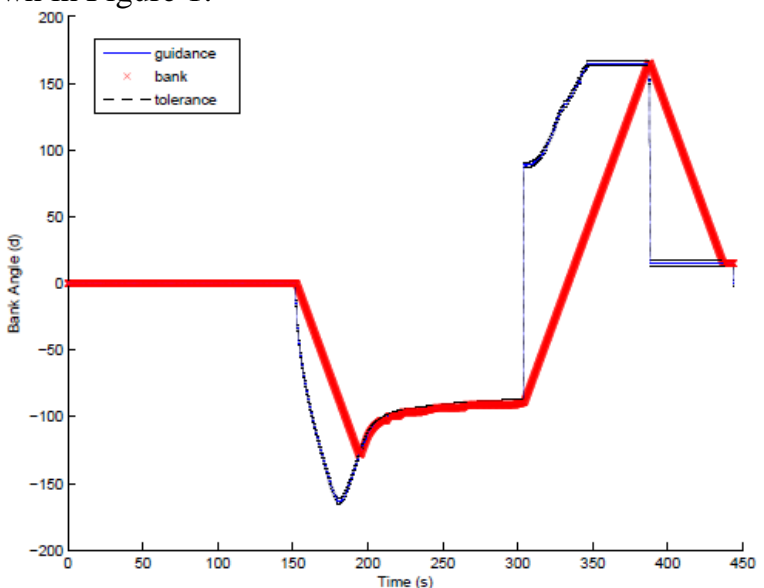

(a) Guidance command and bank angle for $3 \mathrm{~d} / \mathrm{s}^{2}$ and $3 \mathrm{~d} / \mathrm{s}$

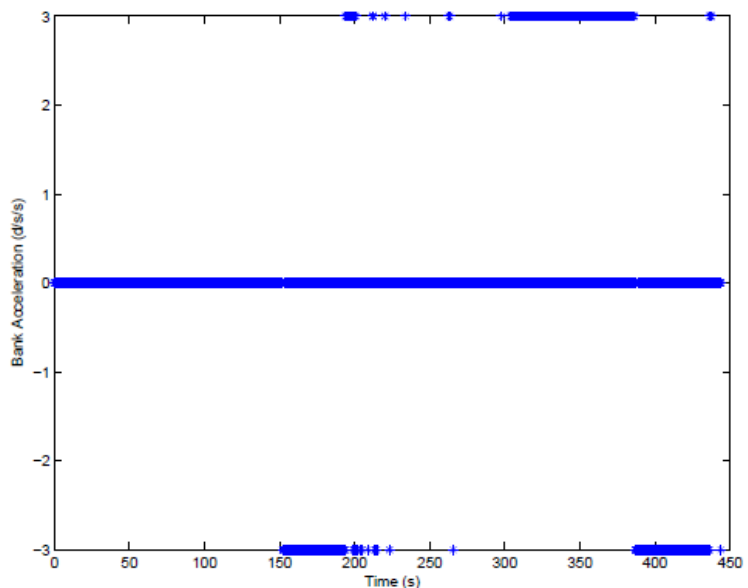

(c) Bank acceleration for $3 \mathrm{~d} / \mathrm{s}^{2}$ and $3 \mathrm{~d} / \mathrm{s}$

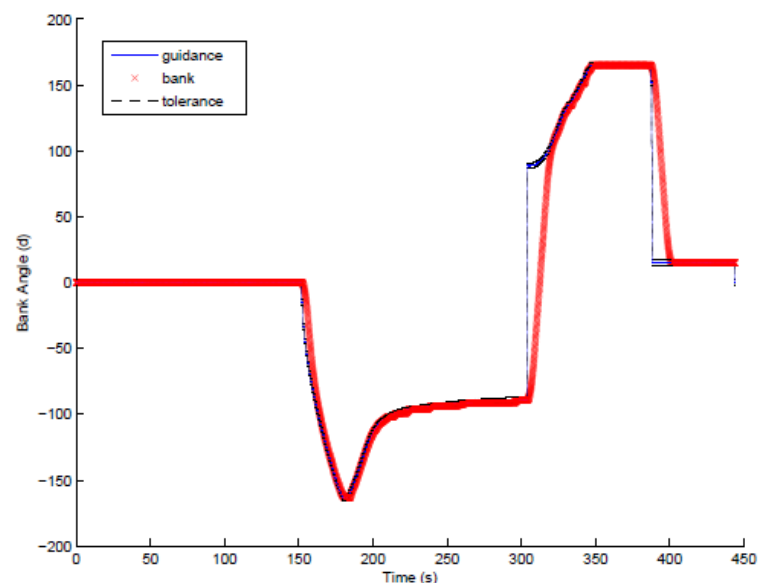

(b) Guidance command and bank angle for $3 \mathrm{~d} / \mathrm{s}^{2}$ and $15 \mathrm{~d} / \mathrm{s}$

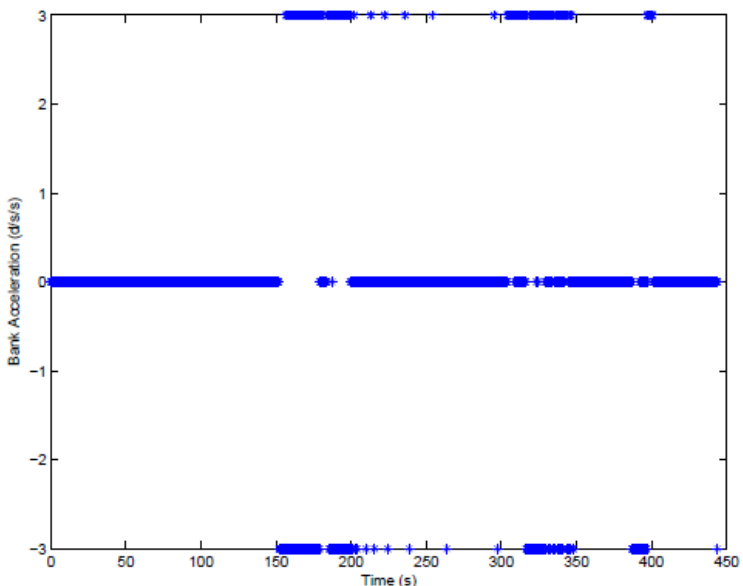

(d) Bank acceleration for $3 \mathrm{~d} / \mathrm{s}^{2}$ and $15 \mathrm{~d} / \mathrm{s}$ 


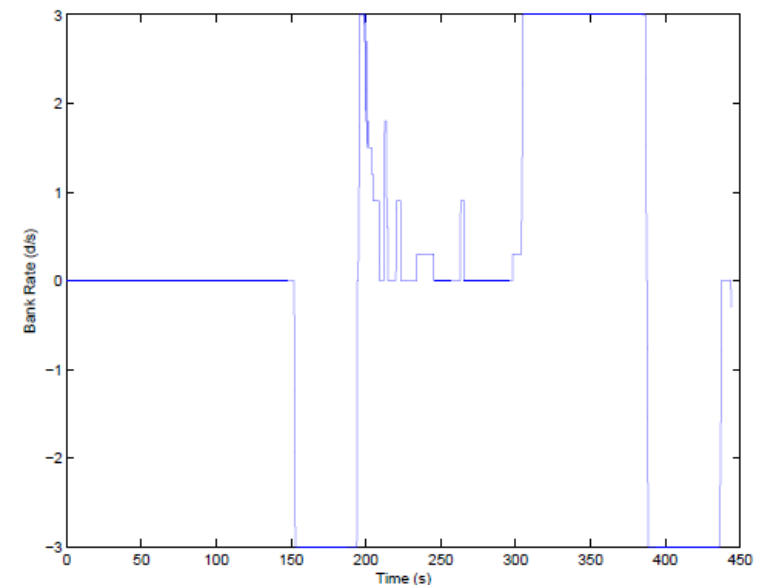

(e) Bank rate for $3 \mathrm{~d} / \mathrm{s}^{2}$ and $3 \mathrm{~d} / \mathrm{s}$

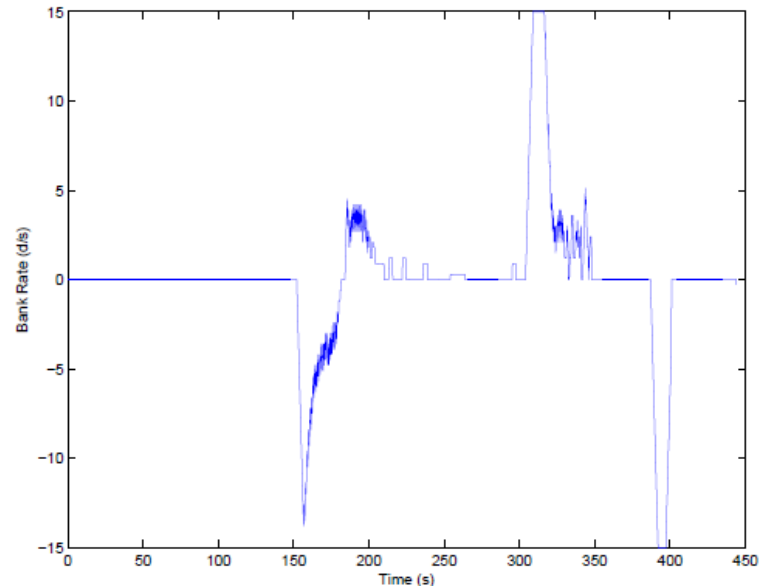

(f) Bank rate for $3 \mathrm{~d} / \mathrm{s}^{2}$ and $15 \mathrm{~d} / \mathrm{s}$

Figure 1. Pseudo-1-DOF controller following commands with a bank acceleration of $3 \mathrm{~d} / \mathrm{s}^{2}$ for bank rate limits $3 \mathrm{~d} / \mathrm{s}$ and $15 \mathrm{~d} / \mathrm{s}$

After producing a nominal pseudo-4-DOF trajectory in FAST, Monte Carlo dispersions were applied to test which combinations of angular acceleration and rate limits are needed before targeting degrades. Bank rates below $10 \mathrm{deg} / \mathrm{s}$ were found to be insufficient for consistent adequate performance, so the study was repeated for bank rate limits of $10-20 \mathrm{deg} / \mathrm{s}$ and accelerations of $1.5-7 \mathrm{deg} / \mathrm{s}^{2}$. The best performance across the tested bank accelerations was achieved with bank rate limits of $15 \mathrm{deg} / \mathrm{s}$ and higher. Results of these studies are shown in Figure 2. Figure 2a shows extreme misses result at very low bank rates of less than $3 \mathrm{deg} / \mathrm{sec}$. Figure $2 \mathrm{~b}$ shows that about $15 \mathrm{deg} / \mathrm{sec}$ is needed for best performance, though it can be slightly lower with a small impact to miss distance.

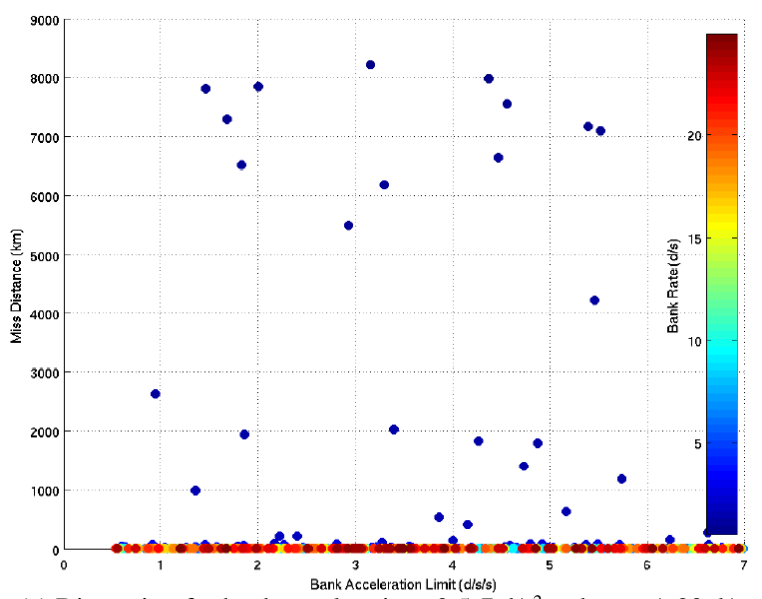

(a) Dispersion for bank accelerations 0.5-7 d/s $\mathrm{s}^{2}$ and rates $1-20 \mathrm{~d} / \mathrm{s}$

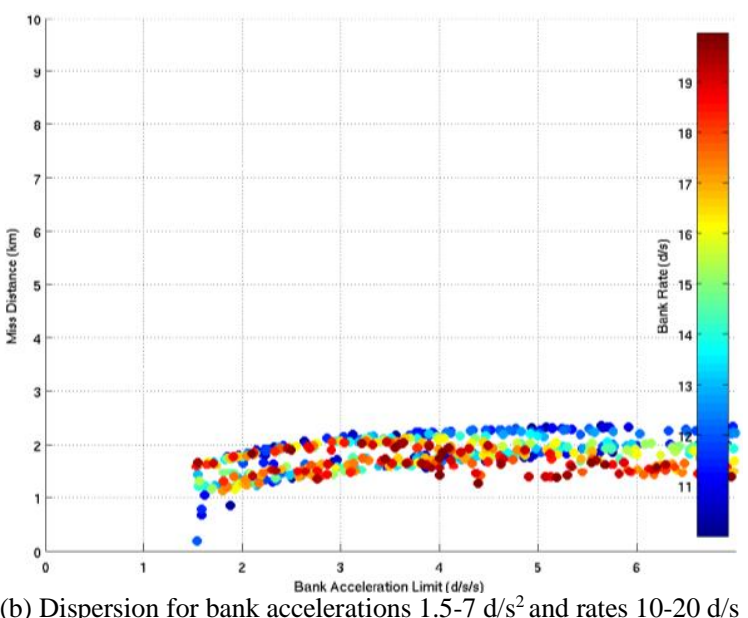

(b) Dispersion for bank accelerations $1.5-7 \mathrm{~d} / \mathrm{s}^{2}$ and rates $10-20 \mathrm{~d} / \mathrm{s}$

Figure 2. Effect of dispersed bank acceleration and rate limits on PDI miss distances

To increase the fidelity of the simulation, a discrete control system needed to be defined based off of the jet sizing study. Locations and orientations of the jets were designed to avoid any direct flow during entry, protect against RCS plume impingement, and ensure that maximum torque arms could be utilized. For these reasons, RCS jets were designed to be placed along the outer perimeter at the aft of the vehicle. While there was a clear minimum bank rate, there was not a clear preference for bank acceleration, so jet locations and orientations were determined for a baseline $5 \mathrm{deg} / \mathrm{s}^{2}$ capability. Previous NASA internal studies on a similar shaped ellipsled vehicle concluded that $5 \mathrm{deg} / \mathrm{s}^{2}$ is best for nominal trajectory performance and contingencies. Minimum total torques needed were calculated for roll, pitch, and yaw axes using the MRV mass properties and thrust accelerations:

$$
\tau_{\text {total }}=I \ddot{\theta}
$$


Assuming a single thruster, the maximum moment arm per axis was combined with these total torques to give the thrust requirements per axis. A notional 4,450 N RCS jet was provided as a study assumption. For the initial vehicle configuration, eight pitch jets were needed to achieve the required torque. Four jets provide pure positive pitch torque and four are used for pure negative pitch torque. The any subset of the jets can be fired during a given cycle, which provides four different levels of pitch torque in both positive and negative pitch. The jets responsible for bank control and sideslip are more complicated in that those eight jets are coupled in yaw and roll. These jets were placed in locations to enable maximum torque but due to the location from the center of gravity (CG), they result in a coupled yaw/roll response. Lastly, four orbital maneuvering jets along the axial direction are included. The primary function of the axial jets is to provide assistance throughout the exo-atmospheric trajectory. However, these jets could also be used for attitude control torques with coupled pitch and yaw moments during the entry phase.

After the discrete jet system was implemented for the nominal trajectory, the jet sizing study was revisited to determine if $5 \mathrm{deg} / \mathrm{s}^{2}$ was indeed adequate for the Mid-L/D. A Monte Carlo analysis was run with a range of thrust levels and confirmed that thrusts below the chosen 4,450 N resulted in poorer performance and higher propellant usage, as shown in Figure 3. Figure 3 shows that a significant loss in landing accuracy occurs at RCS jet thrust levels below $4,000 \mathrm{~N}$.

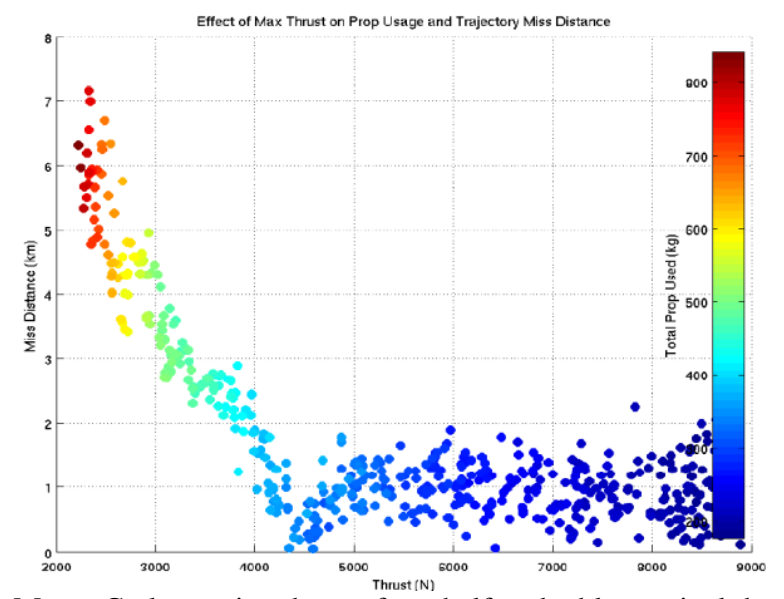

Figure 3. Monte Carlo varying thrusts from half to double nominal thrust values.

Once initial conditions such as atmospheric properties, aerodynamics, and entry state parameters were dispersed for the 4,450 N RCS thrusters in a 6-DOF Monte Carlo simulation, an additional eight jets were needed for adequate performance. The additional jets are 2,225 N, which is half the thrust of the original set of 20 jets. They provide finer resolution in pitch and yaw and result in much improved control performance, as will be shown in subsequent sections. The finalized 28 jet control system is shown in Figure 4.
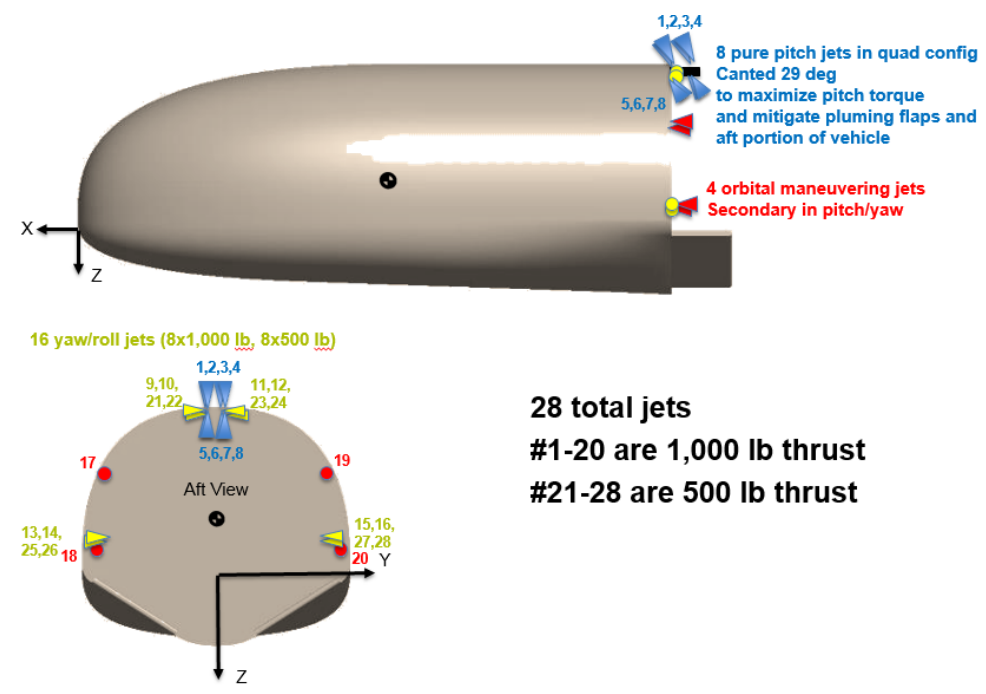

28 total jets

\#1-20 are 1,000 lb thrust

\#21-28 are 500 lb thrust

Figure 4. Finalized RCS Jet Locations. 


\section{II.B. Aerodynamic Static Stability Requirements}

During entry, the control system may exploit a vehicle's natural stability to decrease the amount of propellant needed by maintaining a natural trim angle of attack and sideslip angle. Thus, understanding a vehicle's stability in various flight conditions can prove very useful in determining how a vehicle would naturally respond to expected and unexpected perturbations. Aero-surfaces can improve or lessen vehicle stability and must also be evaluated.

\section{Natural Aerodynamic Static Stability}

Natural stability trim points are defined based on the location of the vehicle's CG and aerodynamic properties. With a CG location of $10.55 \mathrm{~m}$ from the nose and $1 \mathrm{~m}$ above the waterline of the SLS payload fairing, the MRV was designed for zero moments when at angle of attack trim $55^{\circ}$ and sideslip angle trim $0^{\circ}$. Aerodynamic moment coefficients for the Mid-L/D were generated at various angles of attack $(\alpha)$ and sideslip angles $(\beta)$ from Mach 12 to 2 using the Cart3D CFD analysis tool. ${ }^{3}$ The moment is transferred from the aerodynamic moment reference center (MRC) at the nose and applied to the CG as shown in Figure 5.

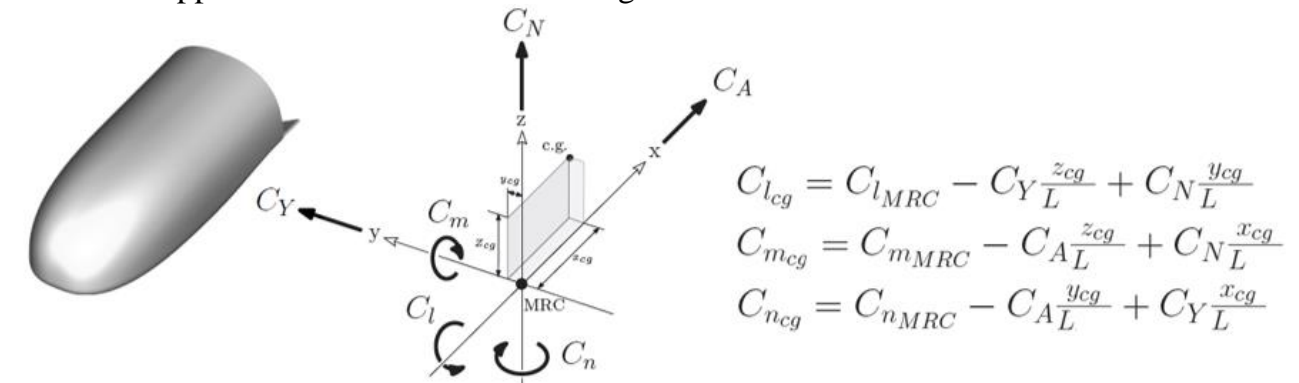

Figure 5. Aerodynamic coordinate system diagram and corresponding moment transfer equations.

Stability derivatives are defined by calculating how these moment coefficients change over various angles of attack, sideslip angles, and Mach numbers and can help a control system predict how the vehicle may respond to an offset from trim conditions at various flight regimes as shown in Equations 4-6.

$$
\begin{aligned}
\tau_{x_{\text {Aero }}} & =C_{l_{\beta, C G}} \beta_{\text {err }} \bar{q} S L \\
\tau_{y_{\text {Aero }}} & =C_{m_{\alpha, C G}} \alpha_{\text {err }} \bar{q} S L \\
\tau_{z_{\text {Aero }}} & =C_{n_{\beta, G G}} \beta_{\text {err }} \bar{q} S L
\end{aligned}
$$

Following the conclusions made from the pseudo-4-DOF study, the next step was to define flight regimes of longitudinal and lateral directional static stability and instability.

Longitudinal aerodynamic stability requires that an increasing pitching moment $\mathrm{Cm}$ corresponds with decreasing angle of attack, leading to a negative $\mathrm{Cm}_{\alpha}$ stability derivative. Across all supersonic Mach numbers sampled, these conditions were met, as shown in Figure 6a. Additionally, the MILSPEC requirements for adequate mission performance and flight safety handling correspond with a vehicle having a short period frequency of at least $1 \mathrm{rad} / \mathrm{s}$ for entry and landing conditions. ${ }^{6}$ This frequency is calculated using the stability derivative and vehicle properties

$$
\omega_{S P}^{2}=-\frac{c_{m_{\alpha}} \bar{q} S L}{I_{y}}
$$

Although the $\mathrm{Cm}_{\alpha}$ was shown to be negative across all Mach numbers, Figure 6b shows that the MILSPEC requirement for natural short period frequency was only met for Mach numbers greater than 8.5 at the trim sideslip angle of $0^{\circ}$. This suggests that a control system would be needed to augment the natural frequency for optimal handling. 


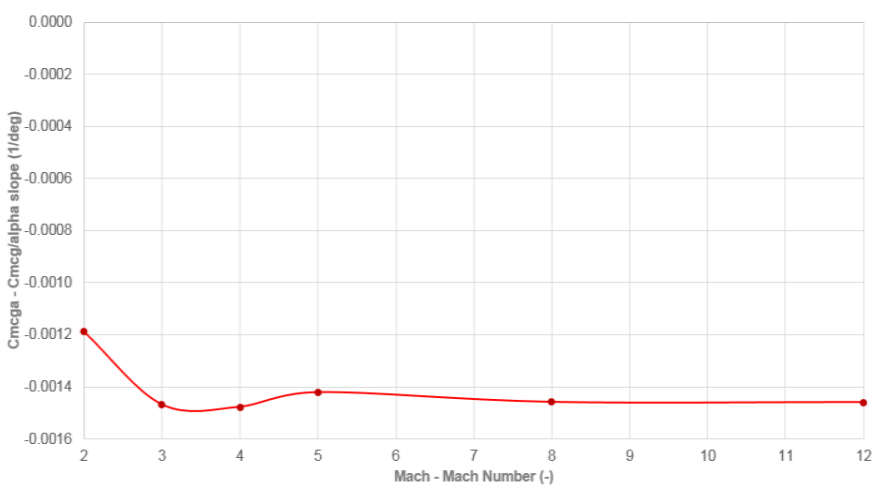

(a) Pitching stability derivative at sideslip trim $0^{\circ}$

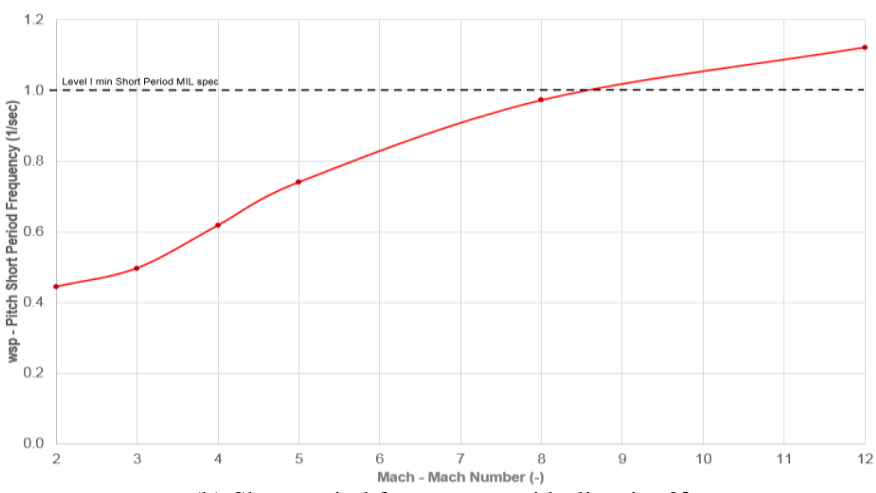

(b) Short period frequency at sideslip trim $0^{\circ}$

Figure 6. Longitudinal stability across supersonic Mach numbers

Lateral aerodynamic stability requires that an increasing rolling moment $\mathrm{Cl}$ corresponds with decreasing sideslip angle, leading to a negative $C l_{\beta}$ stability derivative. Conversely, directional aerodynamic stability requires that an increasing yawing moment $\mathrm{Cn}$ corresponds with increasing sideslip angle, leading to a positive $\mathrm{Cn}_{\beta}$ stability derivative. Similar to airplanes, the Mid-L/D's elongated shape indicates yaw/roll coupling, and as such, lateral directional stability must be evaluated simultaneously. When positive, $C n_{\beta_{d y n}}$ is a parameter used to indicate overall lateral directional static stability at a given angle of attack.

$$
C_{n_{\beta_{d y n}}}=C_{n_{\beta}} \cos \alpha-C_{l_{\beta}} \sin \alpha\left(\frac{I_{z}}{I_{x}}\right)
$$

Figure 7 shows overall lateral directional stability across all Mach numbers generated due to the negative $C_{l_{\beta}}$, positive $C n_{\beta}$, and positive $C_{n_{\beta_{d y n}}}$. MILSPEC requirements for adequate mission performance and flight safety handling corresponds with a vehicle having a Dutch roll frequency of at least $0.4 \mathrm{rad} / \mathrm{s}$ for entry and landing conditions. ${ }^{6}$ This frequency is calculated using the stability derivative and vehicle properties:

$$
\omega_{D R}^{2}=-\frac{{ }_{n_{\beta_{d y n}}} \bar{q} S L}{I_{z}}
$$

The Dutch roll frequency is greater than $0.4 \mathrm{rad} / \mathrm{s}$ and meets level I MILSPEC requirements without augmentation across all Mach numbers at the trim angle of attack of $55^{\circ}$ as also shown in Figure 7.

Like the longitudinal axis, the lateral directional stability derivatives were shown to be stable across all Mach numbers but also naturally met the MILSPEC natural Dutch roll frequency requirements for all Mach numbers at the trim angle of attack. This suggests that without a lateral directional control system, the vehicle would not need augmentation for optimal handling and stability. However, since off-nominal conditions are anticipated and guidance bank commands are necessary for adequate trajectory performance, a control system is still required.

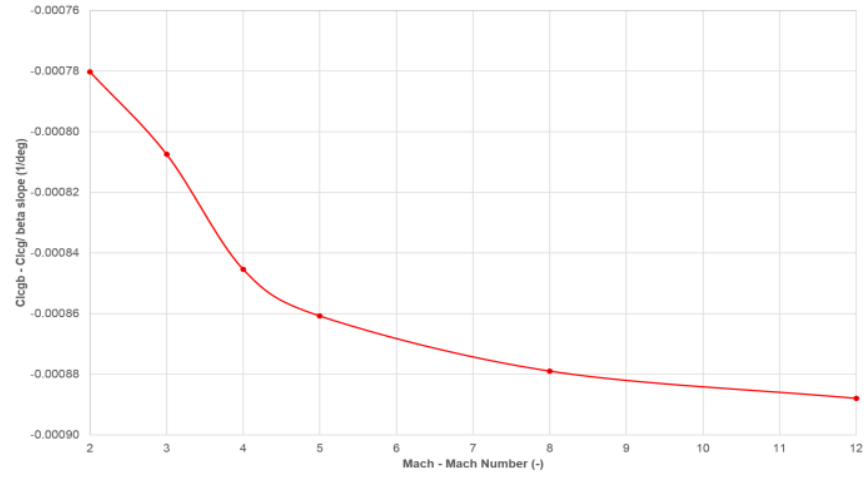

(a) Rolling stability derivative at angle of attack trim $55^{\circ}$

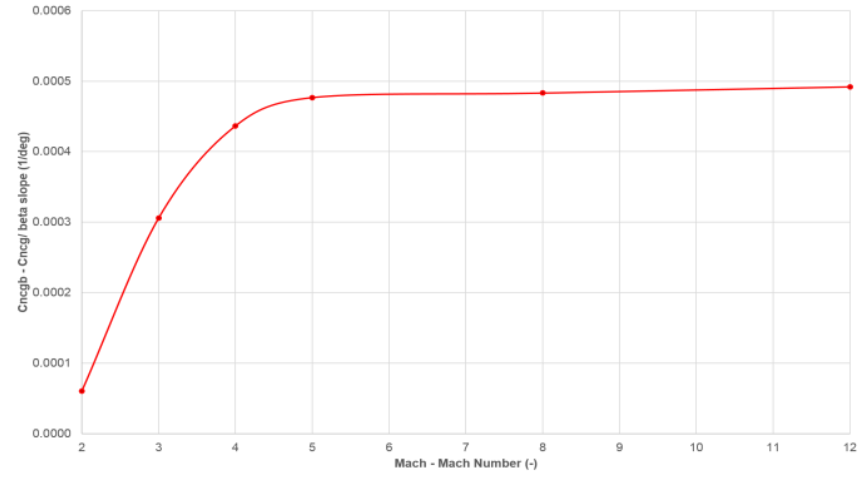

(b) Yawing stability derivative at angle of attack trim $55^{\circ}$ 


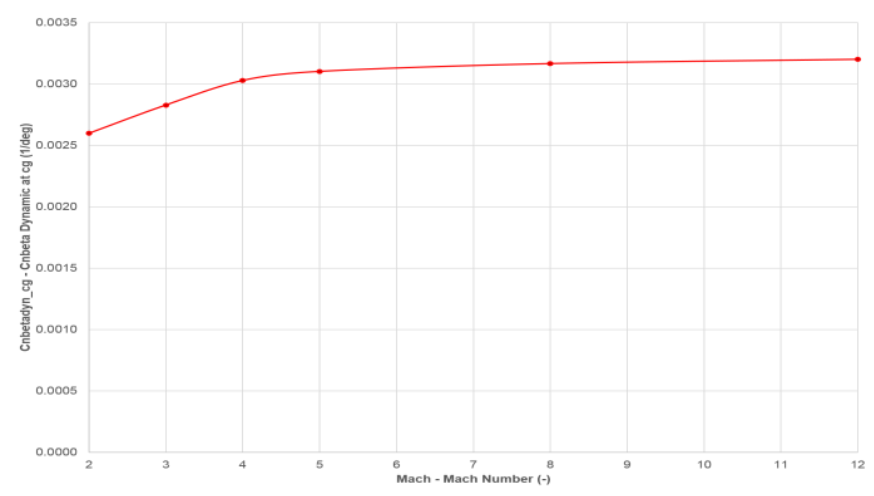

(c) $C n_{\beta_{d y n}}$ at angle of attack trim $55^{\circ}$

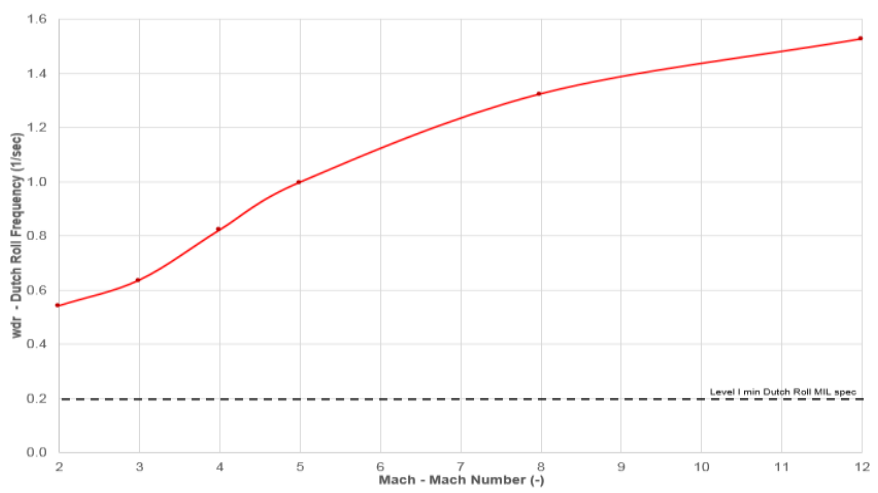

(d) Dutch roll frequency at angle of attack trim $55^{\circ}$

Figure 7. Lateral directional stability across supersonic Mach numbers

\section{Aerosurface Augmentation and CG Uncertainties}

Aerosurfaces such as ailerons, rudders, and elevators have proven to be effective actuators at trimming and reducing propellant usage. For this reason, a split flap is included to assist in angle of attack/sideslip angle trim management and major bank maneuver support during supersonic entry. These flaps can provide longitudinal trim as an elevon or lateral directional trim and bank control as an aileron/rudder. During the vehicle design process, the flaps were sized to provide a sufficient trim capability in the event of CG offsets after vehicle fabrication and cargo loading. Any offset in CG or incorrect estimation of aerodynamic coefficients can introduce a persistent moment at trim conditions, which can be counteracted by aerosurface induced moments. Experience from the Shuttle orbiter influenced the decision to design the controls system and flaps to be capable of managing CG errors of $\pm 20 \mathrm{~cm}$ along the $X$ axis, $\pm 5 \mathrm{~cm}$ along the $Y$ axis, and $\pm 20 \mathrm{~cm}$ along the $Z$ axis. Similar to Shuttle Orbiter, this defined CG box is currently based on percentages of body dimensions. Before implementing these aerosurfaces in the simulation, aerosurface effects on static stability were examined to ensure that they are able to provide adequate trim over this CG box.

Aerodynamic moment coefficients for the MRV's flaps were generated at various angles of attack and sideslip angle conditions using the Cart3D CFD analysis tool. Stability derivatives for the elevon and aileron are defined by calculating how these moment coefficients change over various angles of attack, sideslip angles, and Mach number conditions. These help a control system approximate how the vehicle may respond to aileron or elevon deflections in various flight regimes as shown in Equations 10-12.

$$
\begin{aligned}
\tau_{x_{A S}} & =C_{l_{\delta a}} \beta \bar{q} S L \\
\tau_{y_{A S}} & =C_{m_{\delta e}} \alpha \bar{q} S L \\
\tau_{z_{A S}} & =C_{n_{\delta a}} \beta \bar{q} S L
\end{aligned}
$$

However, the FAST simulated aerodynamic environment does not use stability derivatives for approximated flap contributions and instead applies the effective force and moment coefficients using tabulated data

$$
\begin{aligned}
C_{A} & =C_{A}[M, \alpha, \beta]+\Delta C_{A_{\text {Flaps }}}\left[M, \alpha, \delta_{e}\right] \\
C_{Y} & =C_{Y}[M, \alpha, \beta]+C_{Y_{\delta_{a}}}[M] \delta_{a} \\
C_{N} & =C_{N}[M, \alpha, \beta]+\Delta C_{N_{F l a p s}}\left[M, \alpha, \delta_{e}\right] \\
C_{l} & =C_{l}[M, \alpha, \beta]+C_{l_{\delta_{a}}}[M] \delta_{a} \\
C_{m} & =C_{m}[M, \alpha, \beta]+\Delta C_{m_{\text {Flaps }}}\left[M, \alpha, \delta_{e}\right] \\
C_{n} & =C_{n}[M, \alpha, \beta]+C_{n_{\delta_{a}}}[M] \delta_{a}
\end{aligned}
$$

As previously shown in Figure 5, CG offsets along the $\mathrm{X}$ axis can be corrected by a given elevon deflection. Nominal elevon deflection at trim angle of attack is $0^{\circ}$, but the elevon has a maximum deflection of $\pm 20^{\circ}$. Analysis of the aerodynamic database suggests that CG offsets of $-10 \mathrm{~cm}$ to $30 \mathrm{~cm}$ can be corrected for this range of deflections. Sensitivity to longitudinal CG offsets are defined by static margins, and since the static margin is much larger than the CG box defined, as shown in Figure 8, strong stability is indicated. Any CG offsets or aerodynamic uncertainties that introduce errors outside of this range would need to be corrected using RCS jets. 
The lateral directional axes are more complicated. Unlike most aircraft, the MRV has been designed to nominally fly at a high angle of attack through entry, meaning that, geometrically, bank maneuvers will need more yawing than rolling moment. And although the vehicle has a strong dihedral and therefore restorative rolling moment, the designed flap orientation also favors a stronger yawing than rolling moment. This fact was confirmed when calculating the Lateral Control Departure Parameter (LCDP):

$$
L C D P=C_{n_{\beta}}-C_{l_{\beta}}\left(\frac{C_{n_{\delta_{a}}}}{C_{l_{\delta_{a}}}}\right)
$$

The LCDP criterion predicts if a positive aileron input will output a positive bank (proverse behavior) or a negative bank (adverse behavior) based on the net aerosurface and natural stability contributions. Adverse behavior indicates control reversals, which is when an aircraft rolls in the opposite direction of the pilot's intention. For the MRV, $C l_{\delta_{a}}$ and $C n_{\delta a}$ are strongly negative leading to a negative LCDP and adverse aileron control. For these reasons, the aileron was instead shown to be more effective when used like a rudder.

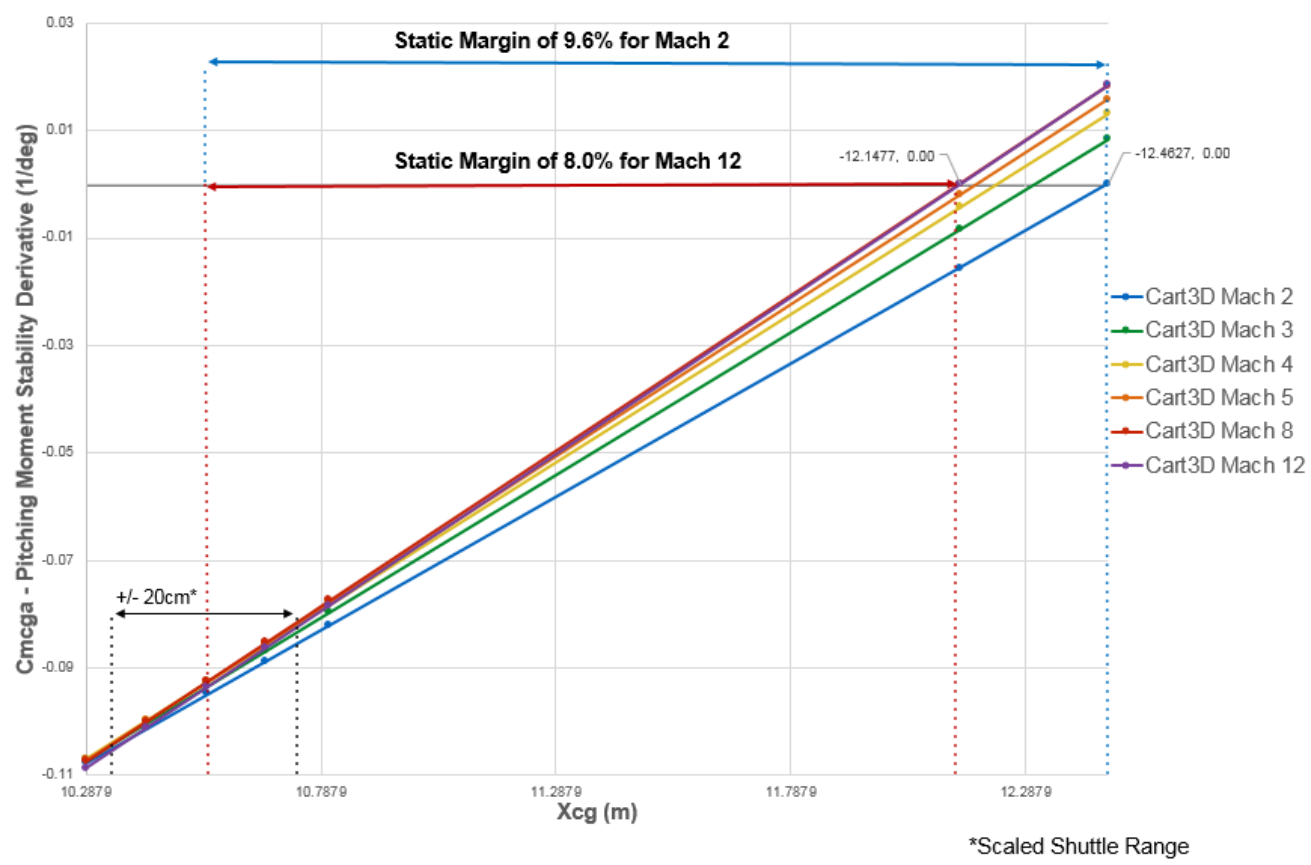

Figure 8. Static margins of $-1.6 \mathrm{~m}$ to $-1.9 \mathrm{~m}$ for Mach 12 to Mach 2

Another complication arises due to the asymmetrical shape of the MRV. This asymmetrical shape indicates that a combination of aileron and sideslip is needed for static stability. This means that if a $\mathrm{Y}_{\mathrm{CG}}$ or $\mathrm{Z}_{\mathrm{CG}}$ offset is introduced, a rudder deflection is not sufficient to completely eliminate any resultant sideslip angle. Nominal aileron deflection at trim sideslip angle is also $0^{\circ}$, and the rudder has a maximum deflection of $\pm 15^{\circ}$. Like aircraft, the MRV has marginally increased stability when the $Z_{\mathrm{CG}}$ is lowered or the $\mathrm{X}_{\mathrm{CG}}$ is moved forward, but has strong sensitivity to any $\mathrm{Y}_{\mathrm{CG}}$ offsets from centerline. Analysis suggests that $Y_{\mathrm{CG}}$ offsets of $\pm 5 \mathrm{~cm}$ can be corrected with combined sideslip angles of $2.5^{\circ}$ to $-4.3^{\circ}$ and aileron deflections of $1^{\circ}$ to $-4^{\circ}$ across all angles of attack and Mach numbers. Combined effects of $Y_{C G}$ and $Z_{\mathrm{CG}}$ offsets can change the natural trim angle of attack through entry and how these relate to the corners of the predefined CG box are shown in Figure 9. Understanding the effects of uncertainties and aerosurface effects on natural static stability, are imperative to interpreting simulation results and analyzing control system performance. 


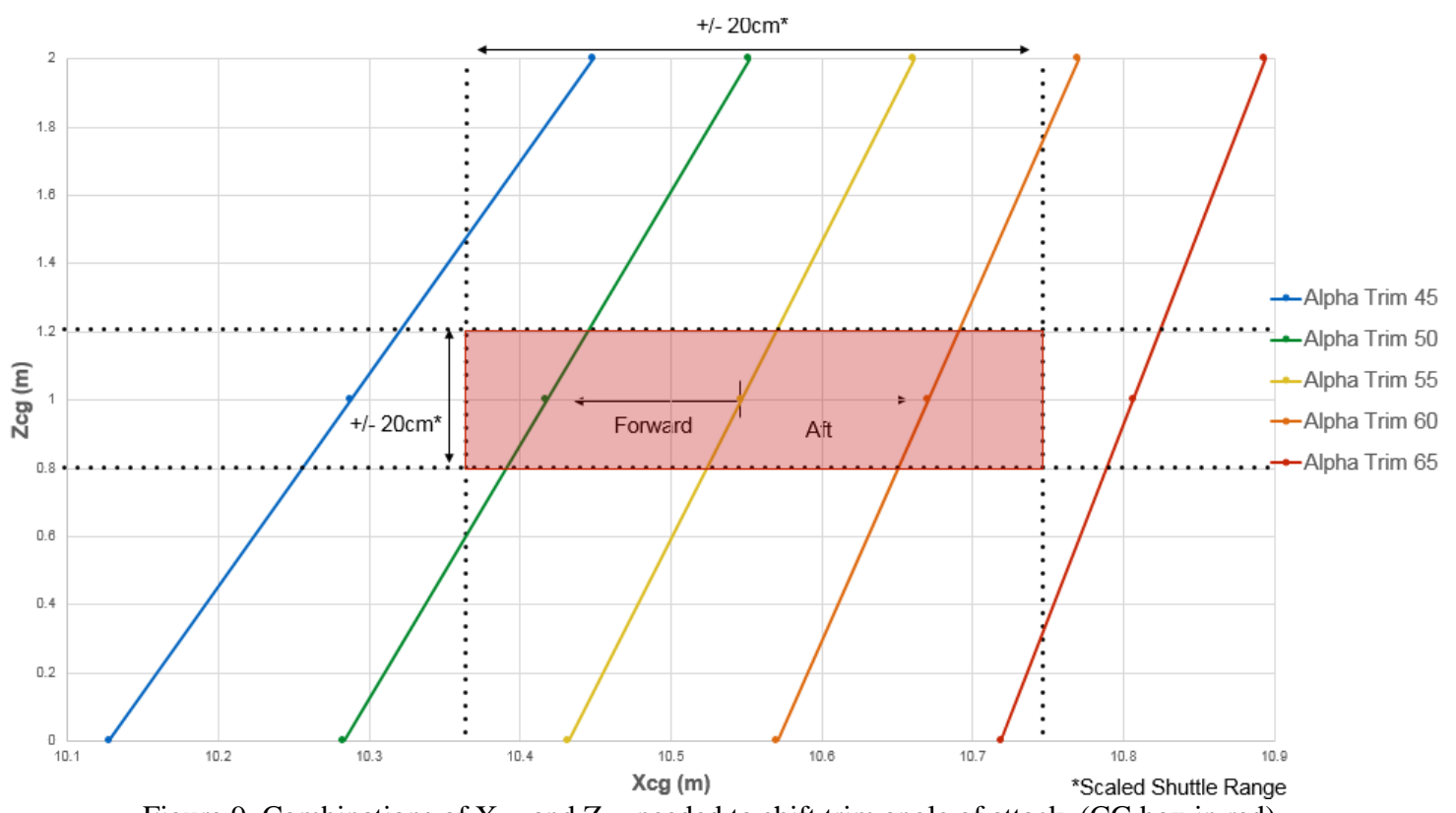

Figure 9. Combinations of $\mathrm{X}_{\mathrm{CG}}$ and $\mathrm{Z}_{\mathrm{CG}}$ needed to shift trim angle of attack. (CG box in red)

\section{II.C. Flight Control System Derivation}

Because the MRV flight control system employs RCS thrusters and aerosurfaces, the derived control system reflects these contributions in the derivation. To reduce complexity and analyze algorithm effectiveness, the first-cut control system only considered RCS jets. Aerosurface contributions were later included. Similar to the AOTV and Shuttle Orbiter, the control system for the MRV was derived by linearizing the nonlinear equations of motion about the expected natural stability trim points. These nonlinear equations are comprised of the expected aerodynamic moments, RCS thruster moments, and aerosurface moments. MILSPEC requirements were used to choose gains that rely on the desired damping ratios and augmented frequencies that the MRV should exhibit for pilot preferential handling.

\section{Assumptions}

Due to the high angle of attack trim and vehicle mass properties, bank error feedback is fed into the yaw axis and coordinated with the roll axis. Pitch error feedback is more simplistic due to its uncoupled nature, and is purely fed into the pitch axis. First the rotational kinematic equations of motion were derived for the freestream, wind, and body frames using a 1-3-2 Euler sequence

where,

$$
\vec{w}^{f b}=\vec{F}_{b} w_{b}^{f b}=\vec{w}^{s b}+\vec{w}^{w s}+\vec{w}^{f w}
$$

$$
\begin{gathered}
\vec{w}^{s b}=\vec{F}_{b} \boldsymbol{w}_{b}^{s b}=\vec{F}_{b}\left[\begin{array}{c}
0 \\
\dot{\alpha} \\
0
\end{array}\right] \\
\vec{w}^{w s}=\vec{F}_{b} \boldsymbol{w}_{b}^{w s}=\vec{F}_{b} \boldsymbol{R}_{b s}(\boldsymbol{\alpha})\left[\begin{array}{c}
0 \\
0 \\
\dot{\beta}
\end{array}\right] \\
\vec{w}^{f w}=\vec{F}_{b} \boldsymbol{w}_{\boldsymbol{b}}^{f w}=\vec{F}_{b} \boldsymbol{R}_{b s}(\boldsymbol{\alpha}) \boldsymbol{R}_{s w}(-\boldsymbol{\beta})\left[\begin{array}{c}
\dot{\phi} \\
0 \\
0
\end{array}\right]
\end{gathered}
$$

and $\vec{F}_{b}$ is a triad of orthonormal dextral vectors defining the body reference frame, also known as a vectrix. ${ }^{7}$ Vectrices are used as a way to differentiate between column matrices composed of scalars, in bold, and the reference frame comprised of unit vectors

$$
\vec{F}_{b}=\left[\begin{array}{l}
\overrightarrow{x_{b}} \\
\overrightarrow{Y_{b}} \\
\overrightarrow{z_{b}}
\end{array}\right]
$$


$\mathbf{R}_{\text {bs }}$ is a rotation matrix about the $\mathrm{Y}$ axis and $\mathbf{R}_{\text {sw }}$ is a rotation matrix about the $\mathrm{Z}$ axis. Thus, the rotational kinematic equations for the MRV resolved in the body frame are expressed as

$$
\boldsymbol{w}_{\boldsymbol{b}}^{\boldsymbol{f b}}=\left[\begin{array}{l}
p \\
q \\
r
\end{array}\right]=\left[\begin{array}{c}
\dot{\phi} \cos \alpha \cos \beta-\dot{\beta} \sin \alpha \\
\dot{\alpha}-\dot{\phi} \sin \beta \\
\dot{\beta} \cos \alpha+\dot{\phi} \sin \alpha \cos \beta
\end{array}\right]
$$

Note that for the linearized control system, rate of change in flight path angle $(\gamma)$ and heading $(\sigma)$ are assumed negligible. Taking the inverse of Equation 18 yields

$$
\left[\begin{array}{c}
\dot{\phi} \\
\dot{\alpha} \\
\dot{\beta}
\end{array}\right]=\left[\begin{array}{c}
p \cos \alpha / \cos \beta+r \sin \alpha / \cos \beta \\
-p \tan \beta \cos \alpha+q-r \tan \beta \sin \alpha \\
p \sin \alpha-r \cos \alpha
\end{array}\right]
$$

These kinematic equations and their assumptions are used when developing the linearized second order differential equations of motion for the MRV with RCS thrusters and aerosurfaces.

\section{RCS Jet Control Logic}

For the initial derivation, a linearized RCS jet model was used where the number of jets commanded is proportional to the error signal. As a way to normalize the gains, a user defined scale factor $\left(\mathrm{m}_{\mathrm{y}}\right)$ was declared, which also denotes the slope of the linear curve of RCS moments to error signal as shown in Figure 10. Each step represents an additional moment contribution from each RCS thruster. The total number of steps, or levels of torque, is denoted by N.

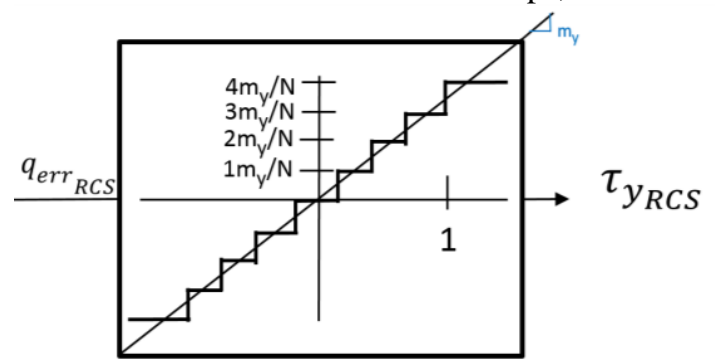

Figure 10. Linearized pitch RCS jet logic

RCS jets will be used for angle of attack trim corrections as well as rate errors, so the desired output torque relies on the equation

$$
\tau_{y_{R C S}}=m_{y}\left(K_{\alpha} \alpha_{e r r}-K_{q} q\right)
$$

Next, the rotational equations of motion for the pitch principal axis is given by

$$
\tau_{y}=I_{y} \dot{q}=C_{m_{\alpha}} \alpha \bar{q} S L+m_{y}\left(K_{\alpha} \alpha_{e r r}-K_{q} q\right)
$$

which includes the previously defined aerodynamic natural static stability derivative. The linearized format of the nonlinear equation of motion is

$$
I_{y} \ddot{\alpha}+k_{\dot{\alpha}} \dot{\alpha}+C_{m_{\alpha}}^{\prime} \bar{q} S L \alpha=0
$$

where $k_{\dot{\alpha}}$ represents the pitching rate gain contribution and $C_{m_{\alpha}}^{\prime}$ is the augmented stability derivative that represents the proportional gain contribution. The assumption is made that at large velocities and trim sideslip angle

$$
\dot{q}=\ddot{\alpha}
$$

which yields

$$
I_{y} \ddot{\alpha}+m_{y} K_{q} \dot{\alpha}-C_{m_{\alpha}} \bar{q} S L \alpha+m_{y} K_{\alpha} \alpha=m_{y} K_{\alpha} \alpha_{c}=\text { constant }
$$

Comparing Equations 22 and 24 results in the definition of the augmented stability derivative

$$
C_{m_{\alpha}}^{\prime}=-C_{m_{\alpha}}+\frac{m_{y} K_{\alpha}}{\bar{q} S L}
$$

In order to transform equation 24 into the desirable format

$$
\ddot{\alpha}+2 \xi \omega_{S P} \dot{\alpha}+\omega_{S P}^{2} \alpha=0
$$


the gains are chosen such that

and

$$
2 \xi \omega_{S P}=\frac{k_{\dot{\alpha}}}{I_{y}}=\frac{m_{y} K_{q}}{I_{y}}
$$

$$
\omega_{S P}=\sqrt{\frac{{ }^{\prime} m_{\alpha} \bar{q} S L}{I_{y}}}
$$

The desired damping ratio and augmented frequencies can be chosen to allow for automatic calculation of gains during simulation runtime at different dynamic pressures. Presumably this automatic gain generation could be replicated using table lookups or velocity measurements real-time in a future flight test. The resultant control system diagram is shown in Figure 11.

Similar to the longitudinal control system derivation, the lateral directional controls also used a linearized RCS jet model where the number of jets commanded is proportional to the error signal for the initial derivation.

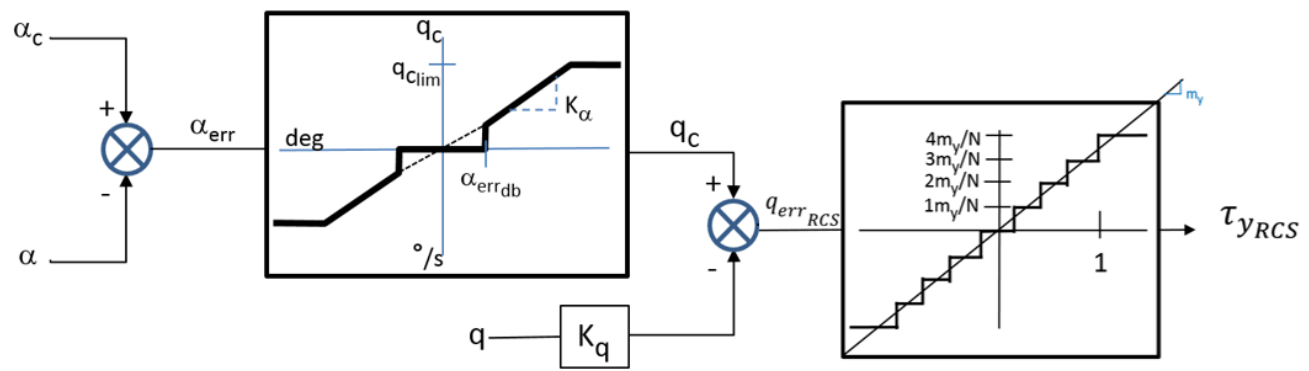

Figure 11. Longitudinal flight control system diagram (RCS only)

As a way to normalize the gains, user defined scale factors $\left(\mathrm{m}_{\mathrm{x}}, \mathrm{m}_{\mathrm{z}}\right)$ were declared as the slopes of the linear curves of roll and yaw RCS moments to error signals as shown in Figure 12. Each step represents an additional moment contribution from each yaw/roll coupled RCS thruster.

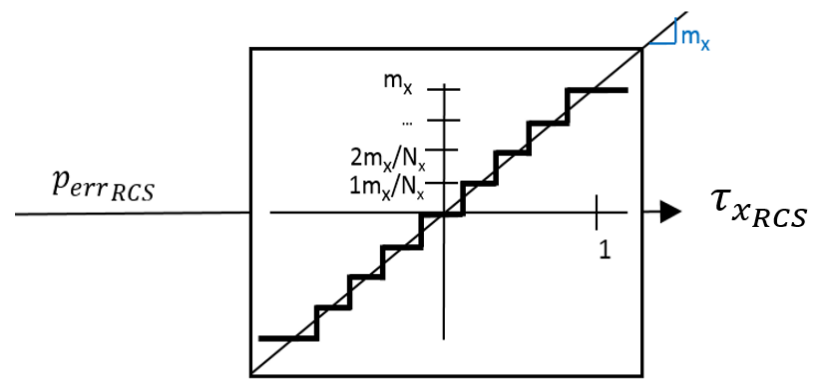

(a) Linearized roll RCS jet logic

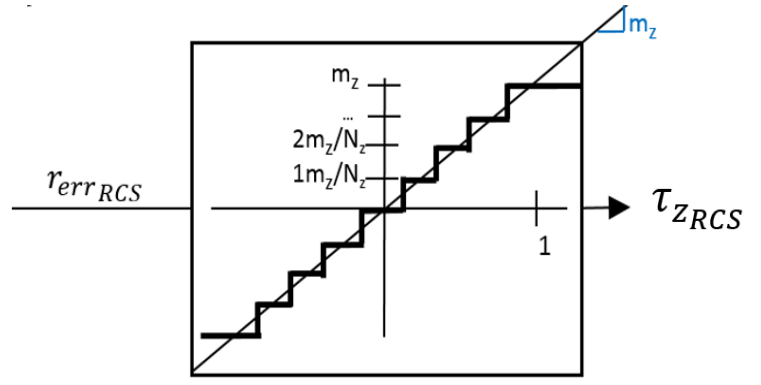

(b) Linearized yaw RCS jet logic

Figure 12. Lateral directional RCS jet logic

RCS jets will be used for sideslip angle trim corrections, bank maneuvers, and bank rate damping, so the desired rotational equations of motion for the roll and yaw principal axis is given by

$$
\begin{aligned}
& \tau_{x}=I_{x} \dot{p}=C_{l_{\beta}} \beta \bar{q} S L+m_{x}\left(-K_{\beta} \beta-K_{p} p+K_{p} r_{c} \operatorname{COT} \alpha\right) \\
& \tau_{z}=I_{z} \dot{r}=C_{n_{\beta}} \beta \bar{q} S L+m_{z}\left(K_{r} r_{c}-K_{r} r\right)
\end{aligned}
$$

which also includes the previously defined aerodynamic natural static stability derivatives. Equations 29 and 30 are a result of the following assumption from Equations 18 and 19

$$
p=r \cot \alpha-\dot{\beta}(\cot \alpha+\sin \alpha), \text { where } \dot{\beta} \approx 0
$$

The linearized format of the nonlinear equation of motion for the lateral direction channel is 


$$
I_{z} \ddot{\beta}+k_{\dot{\beta}} \dot{\beta}+C_{n \beta_{d y n}}^{\prime} \bar{q} S L=\text { constant }
$$

where $k_{\dot{\beta}}$ represents the pitching rate gain contribution and $C_{n \beta_{d y n}}^{\prime}$ is the augmented stability derivative that represents the proportional gain contribution. The assumption is made that at large velocities and trim angle of attack

$$
\ddot{\beta}=\dot{p} \sin \alpha-\dot{r} \cos \alpha, \text { where } \dot{\alpha} \approx 0
$$

Since there are two controllable axes in the lateral directional channel, Equations 29 and 30 are combined with the assumption from Equation 32 to yield

$$
I_{z} \ddot{\beta}+\frac{I_{z}}{I_{x}} \sin \alpha m_{x} K_{p} p+C_{n_{\beta d y n}}^{\prime} \bar{q} S L \beta-m_{z} K_{r} r \cos \alpha=r_{c} \cos \alpha\left(\frac{I_{z}}{I_{x}} m_{x} K_{p}-K_{r} m_{z}\right)=\text { constant }
$$

Comparing Equations 31 and 33 results in the definition of the augmented stability derivative

$$
C_{n \beta_{d y n}}^{\prime}=C_{n_{\beta}} \cos \alpha+m_{x} \frac{I_{z} \sin \alpha K_{\beta}}{I_{x} \bar{q} S L}-C_{l_{\beta}} \frac{I_{z}}{I_{x}} \sin \alpha
$$

In order to transform equation 33 into

$$
\ddot{\beta}+2 \xi \omega_{D R} \dot{\beta}+\omega_{D R}^{2} \beta=\text { const }
$$

the gains are chosen such that

and

$$
2 \xi \omega_{D R}=\frac{m_{x} K_{p}}{I_{x}}=\frac{m_{z} K_{r}}{I_{z}}
$$

$$
\omega_{D R}=\sqrt{\frac{C^{\prime} n_{\beta d y n} \bar{q} S L}{I_{z}}}
$$

Similar to the longitudinal axis, desired damping ratio and augmented frequencies can be chosen to allow for automatic calculation of gains during simulation runtime for different dynamic pressures. Presumably this automatic gain generation could be replicated using table lookups or velocity measurements real-time in a future flight test. The resultant control system diagram is shown in Figure 13. The diagram highlights that the yaw axis controller is primarily used for bank angle modulation, while the roll axis coordinates bank angles and reduces sideslip angle.

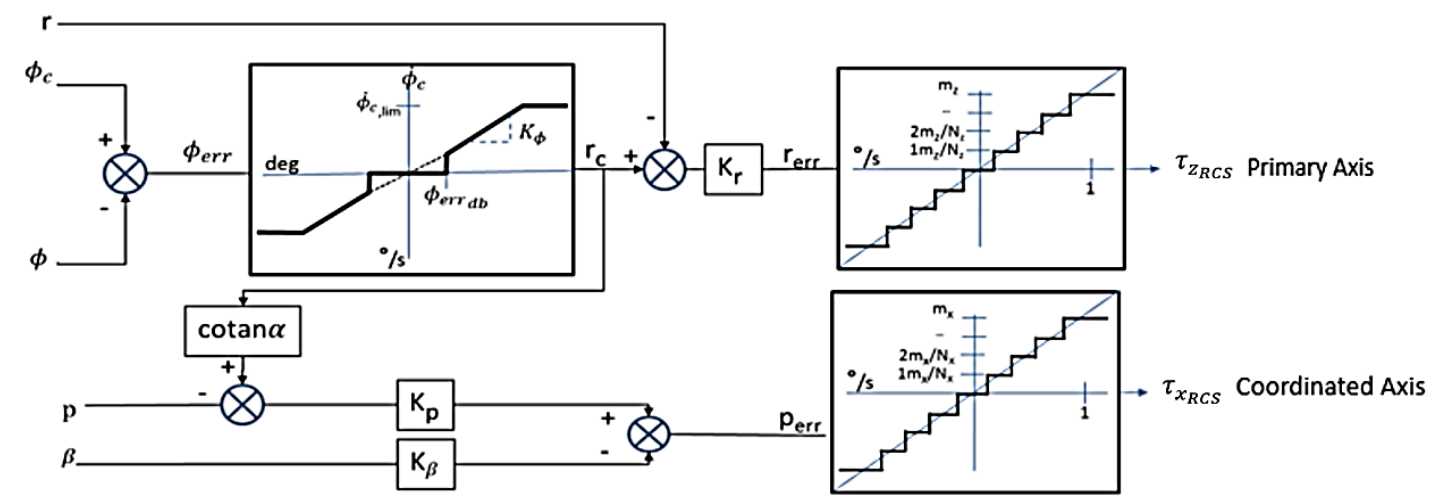

Figure 13. Lateral directional flight control system diagram (RCS only)

As aforementioned, the MILSPEC provides specifications for different levels of flying qualities and was considered when developing the controls system. These specifications address a minimum condition that must be met for a given level of ability to successfully complete a mission with minimal increase in pilot workload. Levels greater than level I correspond to flying characteristics with degradation in mission effectiveness or increase in pilot workload, so it is preferable that the MRV stay in range of the level I defined augmented frequencies. The longitudinal channel requires a short period frequency of $0.5 \mathrm{rad} / \mathrm{s}-3.0 \mathrm{rad} / \mathrm{s}$ for entry and $0.9 \mathrm{rad} / \mathrm{s}-3.0 \mathrm{rad} / \mathrm{s}$ for landing. ${ }^{6}$ The lateral directional channel simply requires a natural Dutch roll frequency of greater than $0.4 \mathrm{rad} / \mathrm{s}$ to meet level I performance for a class III vehicle for entry and landing. A damping ratio of 0.7 was chosen for all versions of the flight control system for fast response with minimal overshoot. This damping ratio also meets MILSPEC requirements. 


\section{RCS Thruster and Aero Surfaces Flight Control System}

After the control system for only RCS actuators was tested in FAST for nominal and dispersed trajectories, aerosurfaces were added to equations of motion to decrease propellant consumption. The aerosurfaces and RCS are used for angle of attack trim corrections and pitching rate damping, so the desired rotational equations of motion are given by

$$
\begin{gathered}
\tau_{x}=I_{x} \dot{p}=C_{l_{\beta}} \beta \bar{q} S L+m_{x}\left(-K_{\beta} \beta-K_{p} p+K_{p} r_{c} \operatorname{COT} \alpha\right)+C_{l_{\delta}} \delta_{a} \bar{q} S L \\
\tau_{y}=I_{y} \dot{q}=C_{m_{\alpha}} \alpha \bar{q} S L+m_{y}\left(K_{\alpha}\left(\alpha_{c}-\alpha\right)-K_{q} q\right)+C_{m_{\delta_{e}}} \delta_{e} \bar{q} S L \\
\tau_{z}=I_{z} \dot{r}=C_{n_{\beta}} \beta \bar{q} S L+m_{z}\left(K_{r} r_{c}-K_{r} r\right)+C_{n_{\delta_{a}}} \delta_{a} \bar{q} S L
\end{gathered}
$$

where $r_{c}=K_{\phi}\left(\phi_{c}-\phi\right)$. Note that with the addition of aerosurfaces, the reduction of the nonlinear equations of motion into their linearized form leaves two equations and one unknown when solving for each of the gains. Unlike the RCS jets, the rudder can only provide support primarily for one axis due to the fact that each deflection results in coupled unequal moments in yaw and roll. The representation of the aerosurfaces is given in terms of the aerosurface gains

$$
\begin{aligned}
& \delta_{e}=K_{\alpha_{A S}}\left(\alpha_{c}-\alpha\right)-K_{q_{A S}} q \\
& \delta_{a}=K_{p_{A S}}\left(r_{c} \cot \alpha-p\right)-K_{\beta_{A S}} \beta
\end{aligned}
$$

Two equations and one unknown led to the decision to handpick constant gains for the aerosurfaces while keeping RCS gain calculations autonomized in the flight control logic. Longitudinal and lateral directional control system diagrams with the addition of the aerosurfaces are shown in blue in Figures 14 and 15. Note that positive elevon deflections yield negative pitch moments.

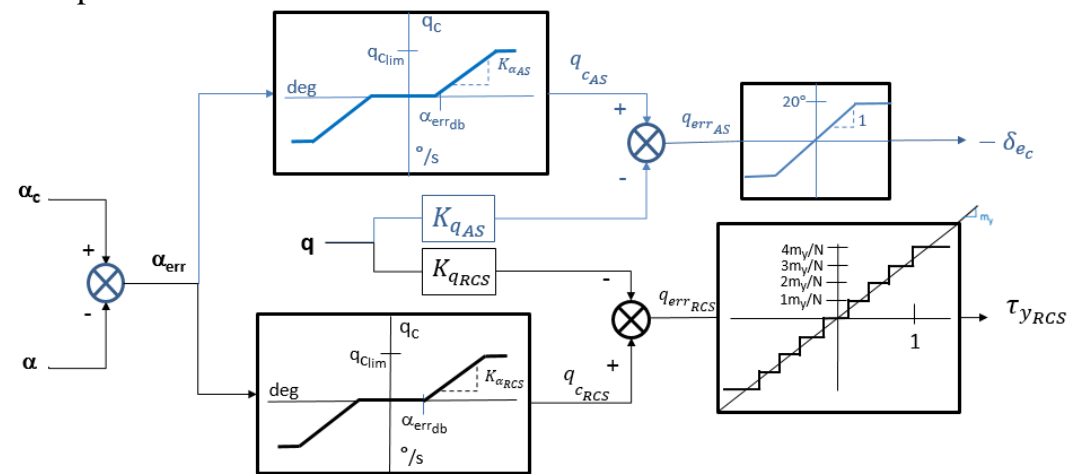

Figure 14. Longitudinal flight control system diagram (RCS and aerosurfaces)

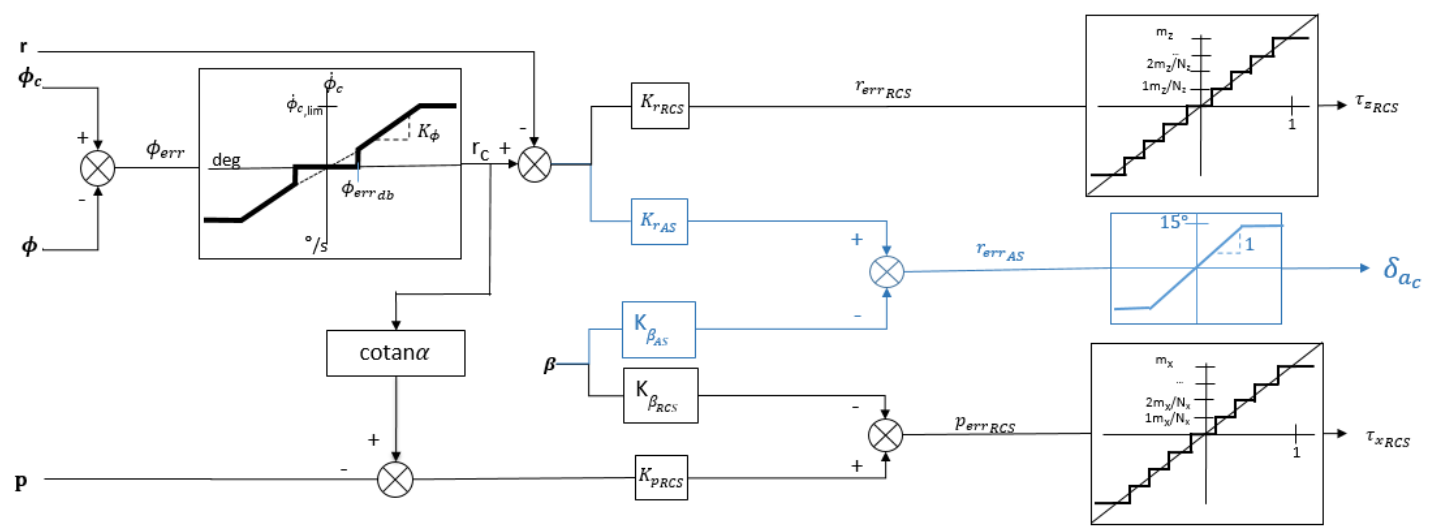

Figure 15. Lateral directional flight control system diagram (RCS and aerosurfaces) 


\section{Control System Performance}

In order to move along a path of increasing fidelity, the MRV entry simulation first employed a 3-DOF perfect controller, followed by a pseudo-1-DOF controller, then linearized RCS jet control system with perfect torque outputs, and finally a discretized RCS jet control system with moving aerosurfaces. The nominal trajectory provided adequate performance in meeting the $5 \mathrm{~km}$ targeting accuracy required at powered descent initiation. It was also imperative that the developed control system be able to replicate the 3-DOF trajectory with minimal degradation in overall performance, which was achieved. Finally the control system needed to withstand dispersions about that nominal trajectory to ensure robustness of the finalized design. The aerosurface and RCS flight control system was able to meet the targeting accuracy required as well as abide by the typical human Mars entry constraints defined in the introduction.

\section{A. Simulation Configuration}

The entry trajectory of the $59 \mathrm{mt}$ MRV was simulated with FAST. The aerodynamic model used a reference length of $20 \mathrm{~m}$ and reference surface area of $160 \mathrm{~m}^{2}$, resulting in a ballistic coefficient of $368 \mathrm{~kg} / \mathrm{m}^{2}$. Initial conditions included a $4.7 \mathrm{~km} / \mathrm{s}$ entry at an inertial flight path angle of $-10.5^{\circ}$ and azimuth of $0^{\circ}$. RCS jets used $4,450 \mathrm{~N}$ of thrust and the aerosurfaces had a maximum deflection angle of $20^{\circ}$ for usage as an elevon and $15^{\circ}$ as a rudder. FNPEG termination point for PDI was set for an energy equivalent of Mach 2 and altitude of $2.8 \mathrm{~km}$. FAST allows for guidance commands and control surfaces to be called at different rates, so FNPEG was called at $1 \mathrm{~Hz}$ and controls at $25 \mathrm{~Hz}$. All results assume a damping coefficient of 0.7 , desired Dutch roll frequency of $2.0 \mathrm{rad} / \mathrm{s}$, and desired short period frequency of $3.5 \mathrm{rad} / \mathrm{s}$. The sideslip angle deadband was $5^{\circ}$ for RCS jets and $4^{\circ}$ for aileron/rudder, but the bank deadband was a more stringent $1^{\circ}$. The angle of attack deadband for RCS was also more stringent to challenge both axes of the control system with a $1^{\circ}$ deadband for RCS jets and $0.5^{\circ}$ for elevon. To increase the fidelity of the simulation, aerosurfaces were restricted to a maximum rate limit of $30 \mathrm{deg} / \mathrm{s}$, based on X-33 experience, while RCS jets received ON/OFF commands.

\section{B. Nominal 6DOF Results}

Desired miss distances of less than $5 \mathrm{~km}$ and max g-loads of less than 4 Earth g's are met by all nominal trajectories. Figure 16 shows agreement between the 3-DOF, pseudo-4-DOF, and linearized RCS jet controlled trajectories modeled in FAST. As a way to assess control system performance, the linearized RCS jet model applied any control system requested torques without coupling or discretization. Once the linearized control system showed agreement, the discrete RCS jets control logic was used along with jet selection logic. The selection logic choses the appropriate jets to provide the requested torques by using a table of jet combinations and their corresponding torques.

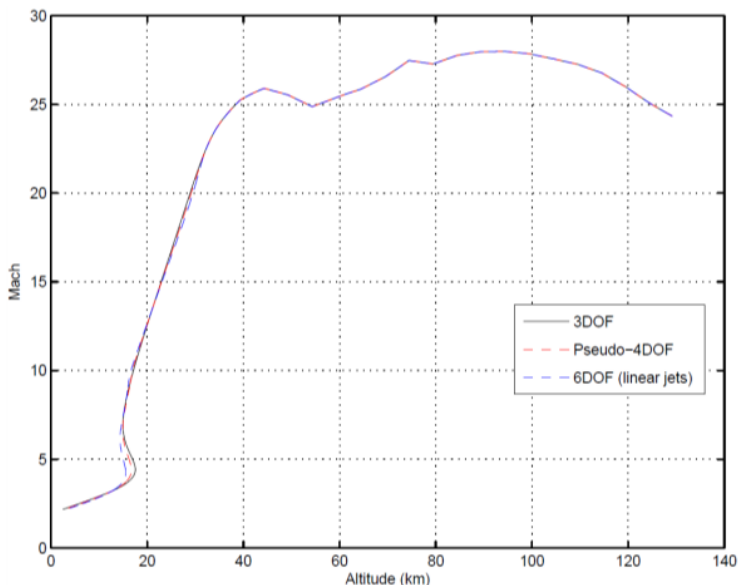

(a) Mach vs. Altitude

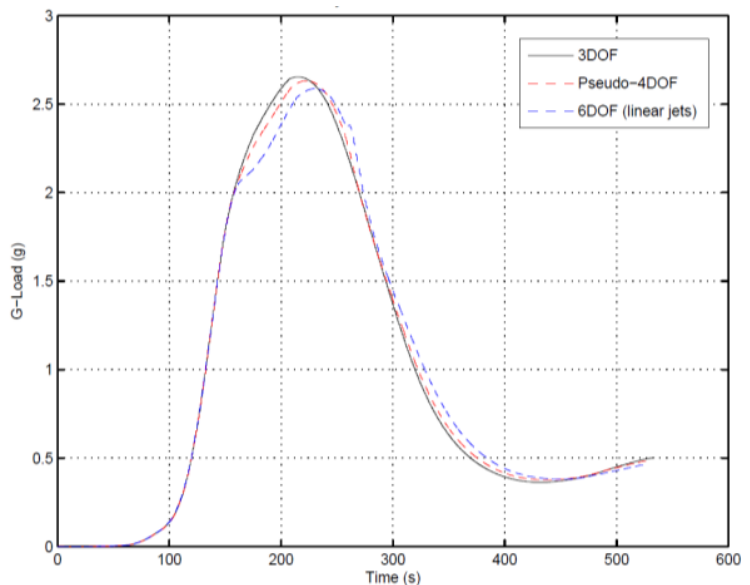

(b) G-load vs. Time 


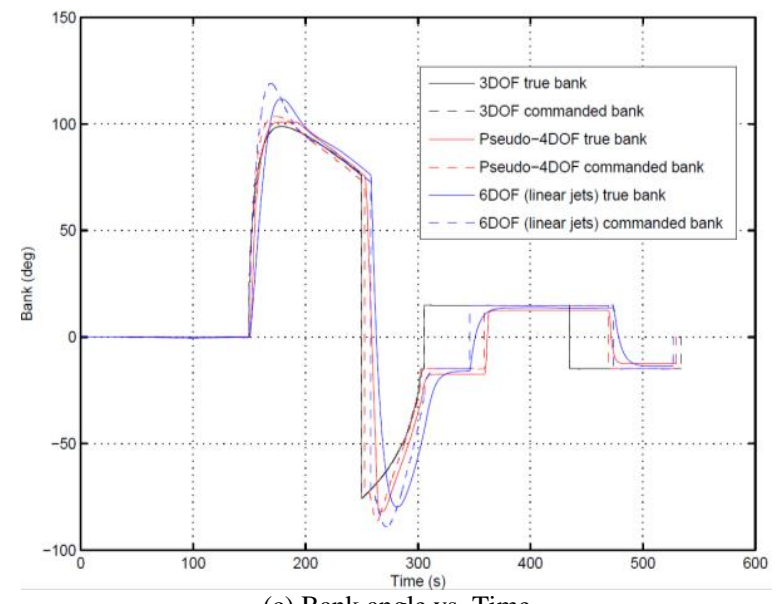

(c) Bank angle vs. Time

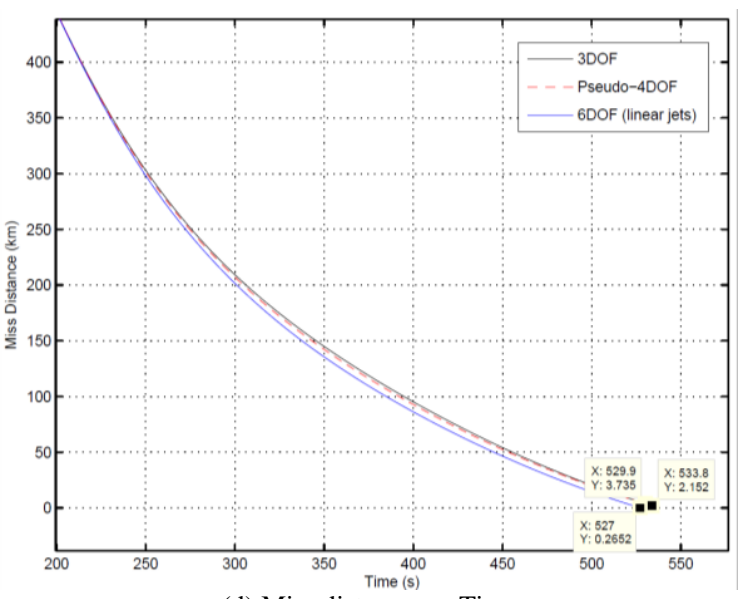

(d) Miss distance vs. Time

Figure 16. Comparison of the increased fidelity nominal trajectories

Figures 17 shows the nominal 6-DOF trajectory with discrete jets and rate limit constrained aerosurfaces. These results also abide by the aforementioned constraints as well as desired control deadbands and rate limits. The pitch channel has an angle of attack deadband of $0.5^{\circ}$ for the elevon and $1^{\circ}$ for the RCS jets. Rates are well below the 15 $\mathrm{deg} / \mathrm{s}$ limit. Elevon becomes more negative with time due to the decreasing angle of attack trim. Pitch jets fire only when angle of attack is outside deadband at low $\bar{q}$ (flaps ineffective) and the large bank reversal at 250s. The yaw/roll channel has a sideslip deadband of $4^{\circ}$ for the aileron and $5^{\circ}$ for the RCS jets. Rates are well below the $15 \mathrm{deg} / \mathrm{s} \mathrm{limit}$ except at the large bank reversal, so rate limits were increased to $20 \mathrm{deg} / \mathrm{s}$ to highlight the trajectory's unconstrained peak rate. Aileron deflections are typically small, primarily tracking the yaw rate and bank commands. Yaw and roll jets fire more frequently to track guidance commands. Sideslip angle remains less than $1^{\circ}$ until the large bank reversal, but still stays within the RCS jet deadband.

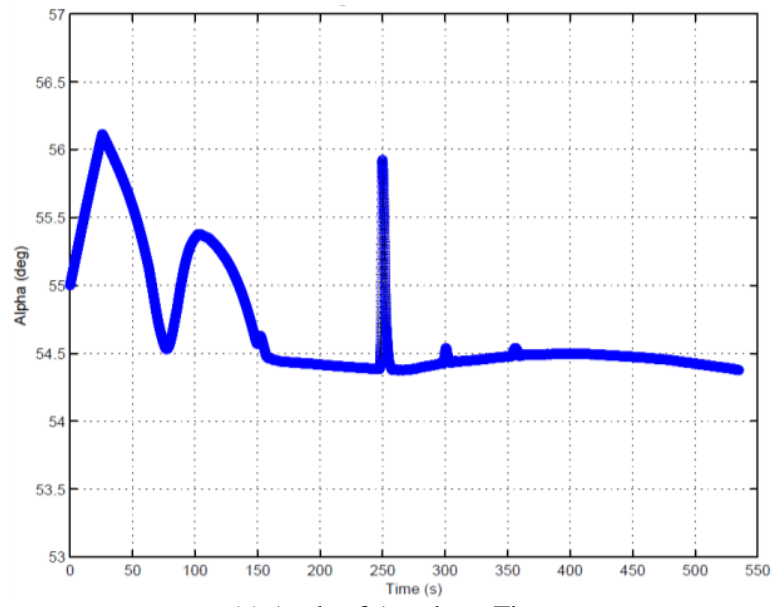

(a) Angle of Attack vs. Time

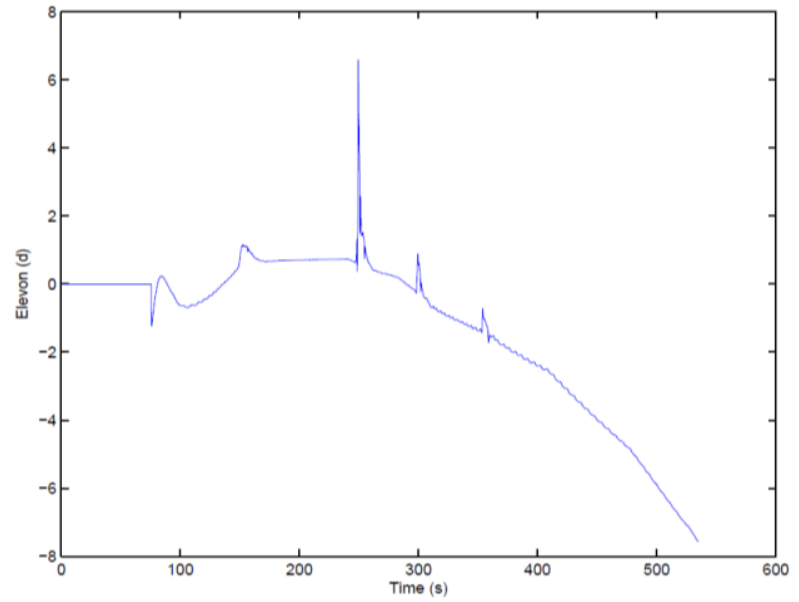

(b) Elevon deflection vs. Time 

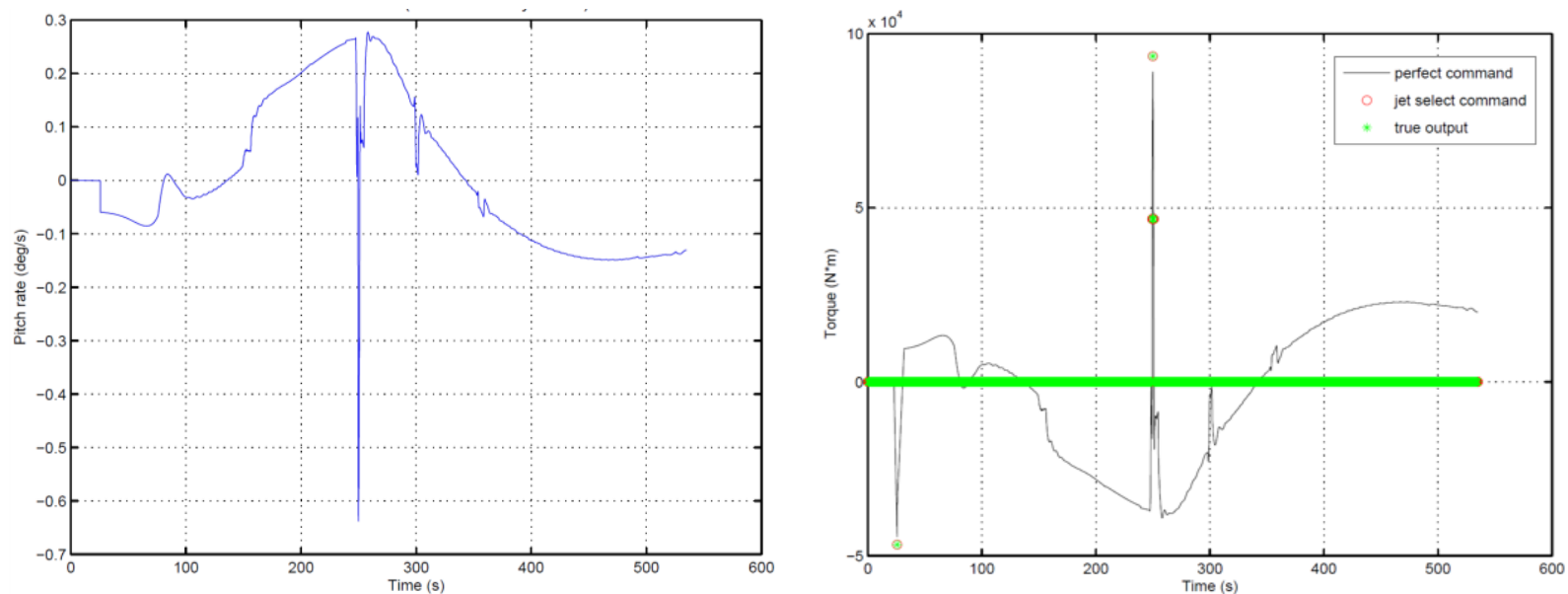

(c) Pitch rate vs. Time

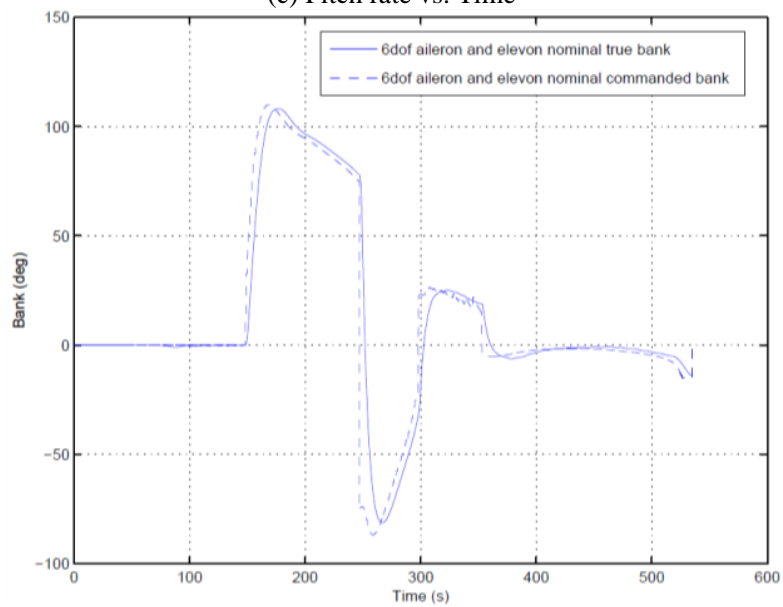

(d) Pitch torque vs. Time

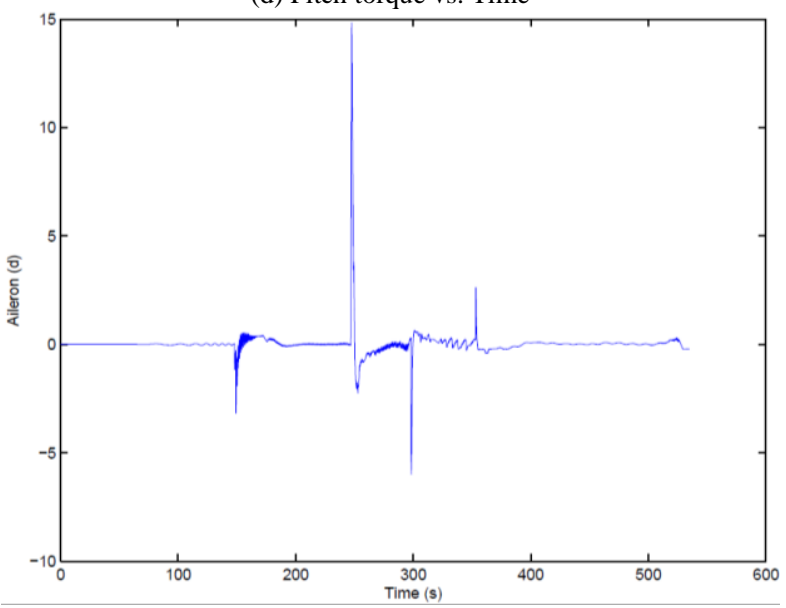

(e) Guided bank angle vs. Time

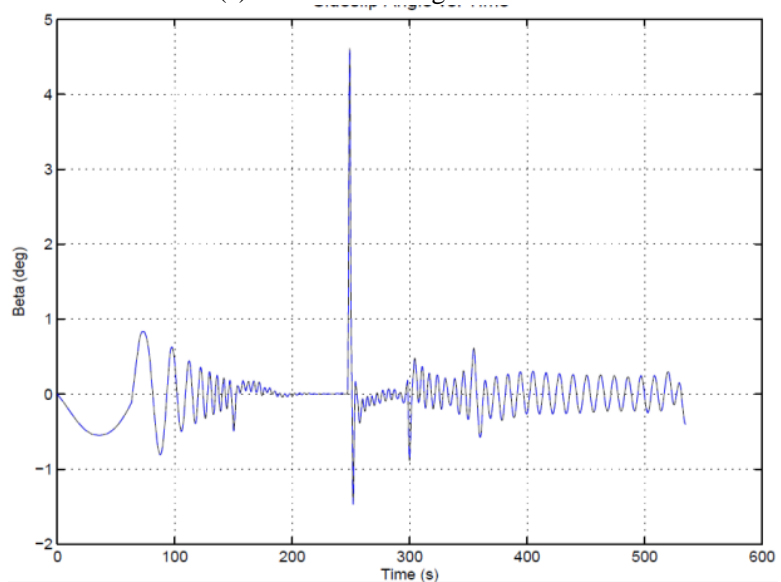

(g) Sideslip angle vs. Time

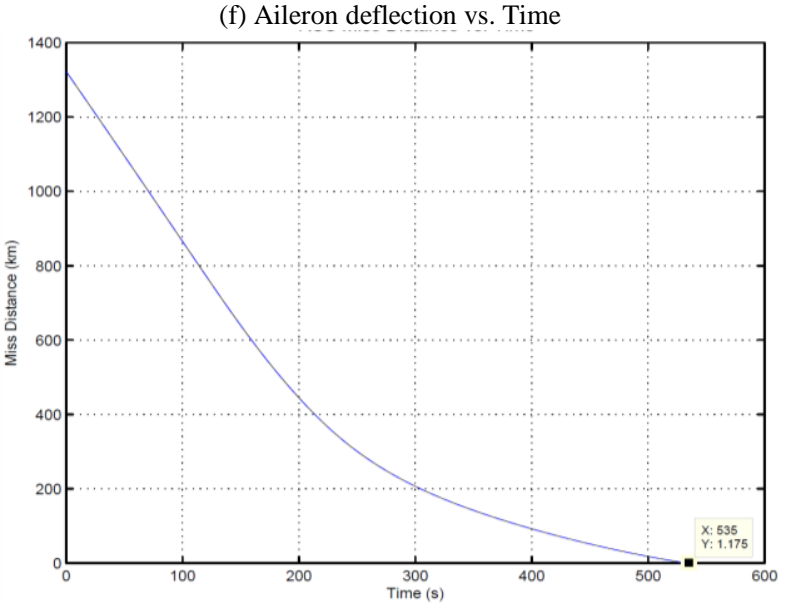

(h) Miss distance vs. Time 


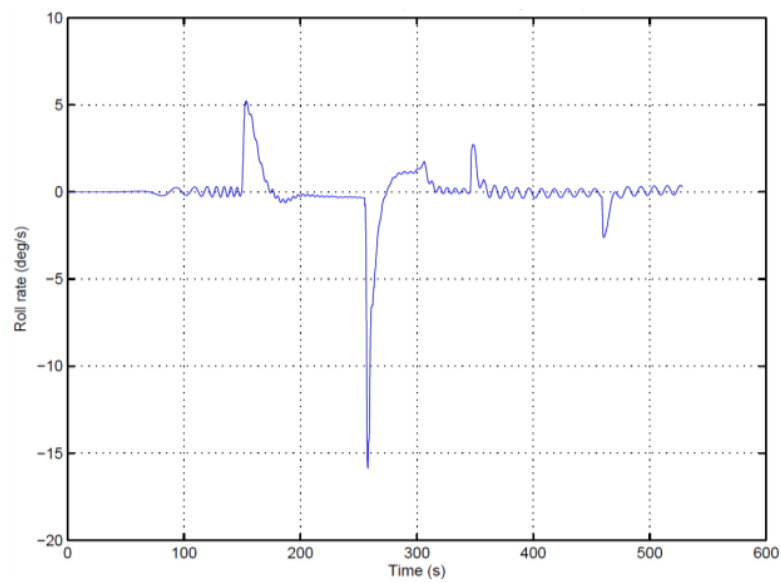

(i) Roll rate vs. Time

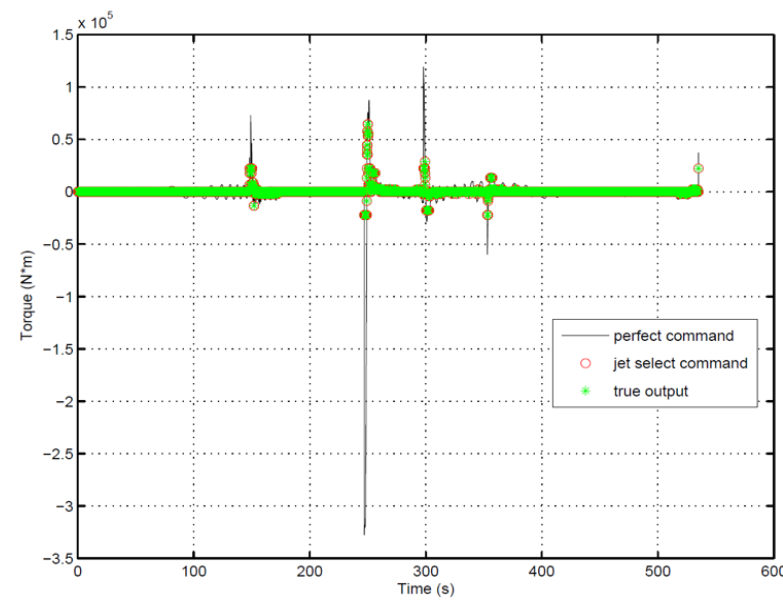

(k) Roll torque vs. Time

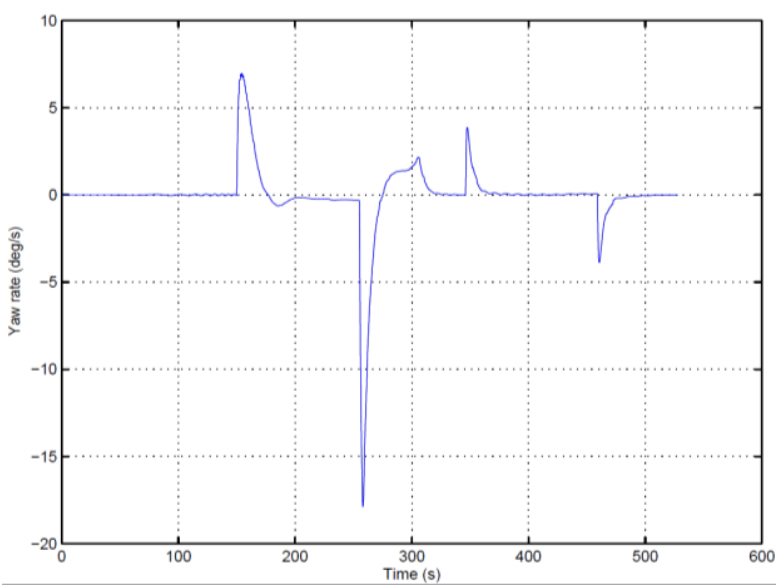

(j) Yaw rate vs. Time

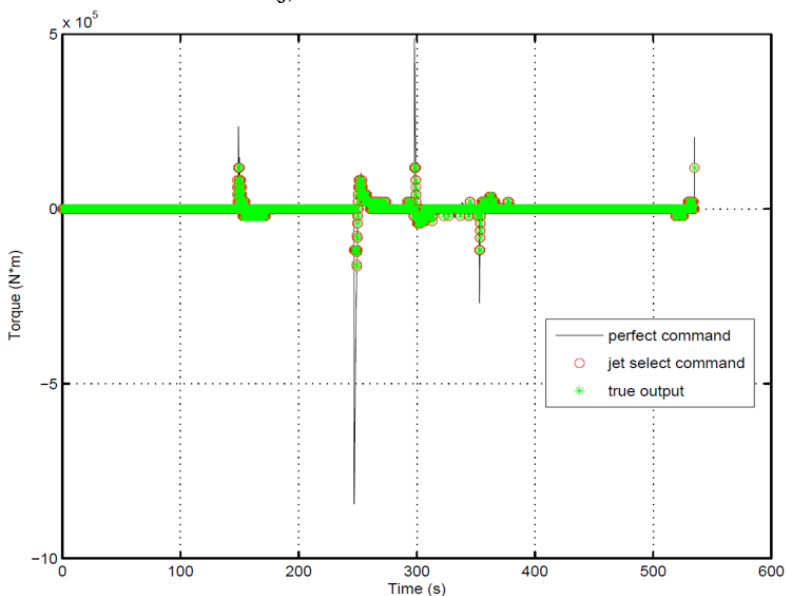

(1) Yaw torque vs. Time

Figure 17. Nominal 6-DOF trajectory (RCS and aerosurfaces)

Torque plots show the linearized jet commands in black, the discrete jet selection logic commands in red, and the true torque outputs in green. Note that the jet selection logic command and true output torques will typically align unless there is a CG offset of which the flight software is unaware. Figure 18 shows a comparison in fuel consumption before and after using flaps. Most savings are due to the highly effective elevon, leaving the majority of prop usage due to large bank maneuvers. Flaps reduced total prop by more than one half.

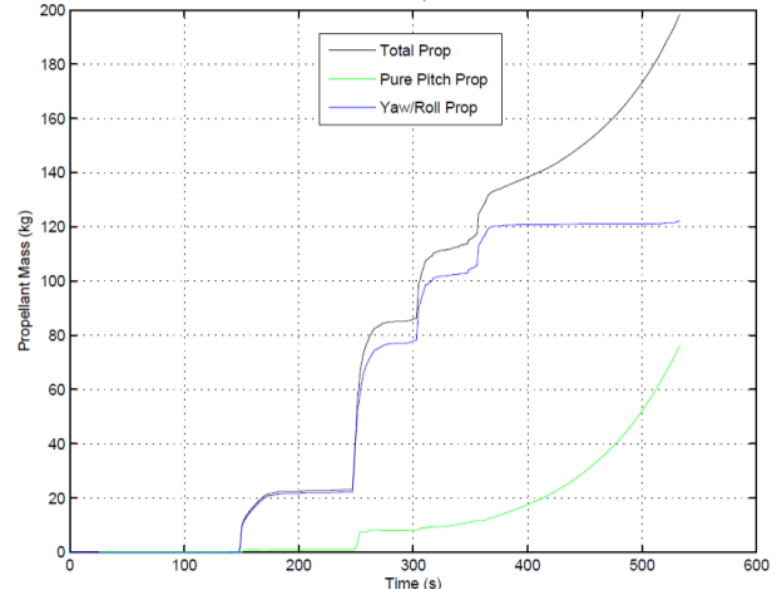

(a) Propellant used vs. Time (RCS only)

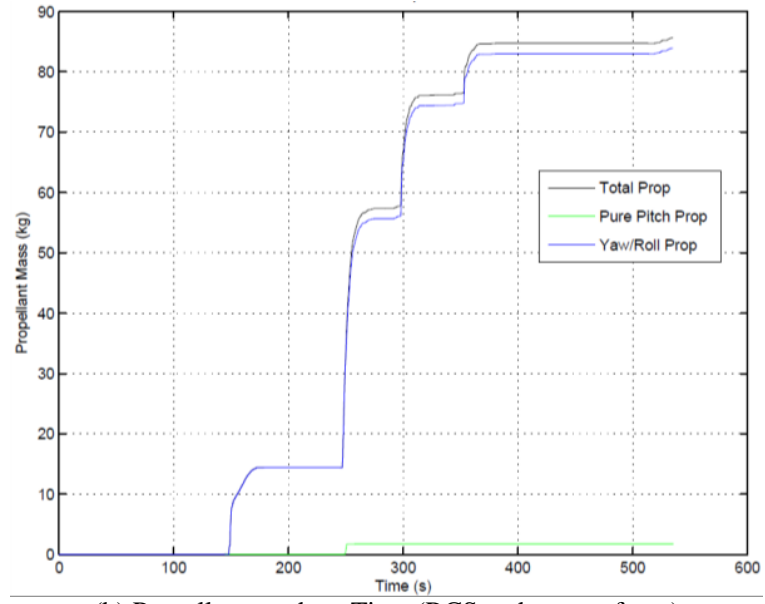

(b) Propellant used vs. Time (RCS and aerosurfaces)

Figure 19. Propellant usage for nominal 6-DOF trajectories 


\section{Conclusions}

Successful nominal and dispersed results imply that a combination of aerosurfaces and RCS jets provide an adequate control system design for the MRV. These results provide a solid first step in the control system design and 6-DOF simulation development efforts for the Mars Pathfinder Study. Lessons learned and assumptions made will provide insight into the considerations and decisions for not only the MRV but other candidates of the study. The aero stability results can help to broaden the general knowledge and understanding of high angle of attack vehicles and operations. Future work includes assessing other possible control logic and jet selection logic algorithms. Bank angle and angle of attack modulation will be further explored to assess the benefits of using another degree of freedom in the guidance control strategy. Monte Carlos results will also need to be generated to assess the robustness of the guidance and control algorithms. This study included no navigation state errors or dispersions, and those must be introduced for a more realistic understanding of vehicle performance. Finally, hardware-in-the-loop testing will help to illuminate any over-simplifications that need to be addressed before a flight test.

\section{Acknowledgements}

The authors would like to extend a thank you to all the parties that have contributed to the MRV 6-DOF simulation efforts. In no particular order, we would like to acknowledge the efforts of Phil Robinson, Chuck Campbell, Edward Robertson, Alicia Dwyer Cianciolo, Tara Polsgrove, Joseph Garcia, and Francis Monahan.

\section{References}

${ }^{1}$ Cianciolo, A. D., Polsgrove, T. T. "Human Mars Entry, Descent, and Landing Architecture Study Overview” SPACE 2016, AIAA 2016-5494.

${ }^{2}$ P. Brugarolas, M. San Martin, E. Wong, The RCS attitude controller for the exo-atmospheric and guided Entry phases of the Mars Science Laboratory, in International Planetary Probe Workshop, Barcelona, Spain (2010)

${ }^{3}$ Ronald R. Sostaric, Christopher J. Cerimele, Edward A. Robertson, and Joseph A. Garcia. "A Rigid Mid Lift-to-Drag Ratio Approach to Human Mars Entry, Descent, and Landing", AIAA Guidance, Navigation, and Control Conference, AIAA SciTech Forum, (AIAA 2017-1898).

${ }^{4}$ Ping Lu, "Entry Guidance: A Unified Method", Journal of Guidance, Control, and Dynamics, Vol. 37, No. 3, 2014, pp. 713728.

${ }^{5}$ Gamble, J.; Spratlin, K.; Skalecki, L. Lateral Directional Requirements for a Low L/D Aeromaneuvering Orbital Transfer Vehicle. AIAA Paper 84-2123 presented at AIAA Atmospheric Flight Mechanics Conference (Seattle, Wash.). Aug. 1984.

6 Anon; MIL-F-8785c, Military Specification Flying Qualities of Piloted Airplanes; Air Force Flight Dynamics Laboratory, Wright-Patterson Air Force Base, Dayton, OH, November 1980.

${ }^{7}$ A. de Ruiter, C. Damaren, J. Forbes, Spacecraft Dynamics and Control - An Introduction, John Wiley and Sons Ltd, January 2013. 